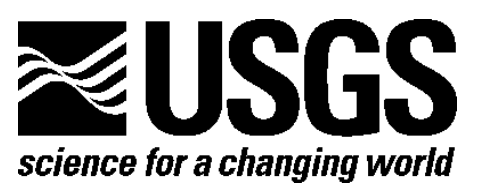

\title{
Advanced Spaceborne Thermal Emission and Reflection Radiometer Level 1 Precision Terrain Corrected Registered At-Sensor Radiance (AST_L1T) Product, Algorithm Theoretical Basis Document
}

By David Meyer, Dawn Siemonsma, Barbara Brooks, and Lowell Johnson

Open-File Report 2015-1171

U.S. Department of the Interior

U.S. Geological Survey 


\section{U.S. Department of the Interior SALLY JEWELL, Secretary}

\section{U.S. Geological Survey \\ Suzette M. Kimball, Acting Director}

U.S. Geological Survey, Reston, Virginia: 2015

For more information on the USGS-the Federal source for science about the Earth, its natural and living resources, natural hazards, and the environment-visit http://www.usgs.gov or call 1-888-ASK-USGS (1-888-275-8747)

For an overview of USGS information products, including maps, imagery, and publications, visit http://www.usgs.gov/pubprod

To order this and other USGS information products, visit http://store.usgs.gov

Any use of trade, firm, or product names is for descriptive purposes only and does not imply endorsement by the U.S. Government.

Although this information product, for the most part, is in the public domain, it also may contain copyrighted materials as noted in the text. Permission to reproduce copyrighted items must be secured from the copyright owner.

Suggested citation:

Meyer, David, Siemonsma, Dawn, Brooks, Barbara, and Johnson, Lowell, 2015, Advanced Spaceborne Thermal Emission and Reflection Radiometer Level 1 Precision Terrain Corrected Registered At-Sensor Radiance (AST_L1T) product, algorithm theoretical basis document:

U.S. Geological Survey Open-File Report 2015-1171, 44 p., http://dx.doi.org/10.3133/ofr20151171.

ISSN 2331-1258 (online) 


\section{Acknowledgments}

The Advanced Spaceborne Thermal Emission and Reflection Radiometer (ASTER) Level 1 Terrain and Precision Corrected Radiance At-Sensor product is generated in collaboration with the National Aeronautics and Space Administration and the U.S. Geological Survey, where time-proven geometric algorithms developed for Landsat precision and terrain corrected products have been adapted for use with ASTER imagery.

We wish to thank James Storey, Pat Scaramuzza, and Donald Moe of Stinger Ghaffarian Technologies Incorporated, contractor to the U.S. Geological Survey, for reviewing this document and providing valuable comments and suggestions.

We would also like to express our gratitude to Ron Morfitt of the U.S. Geological Survey for his contributions. 


\section{Contents}

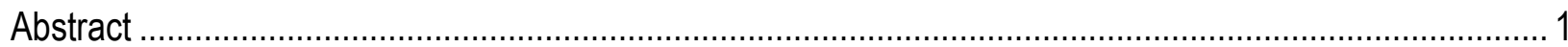

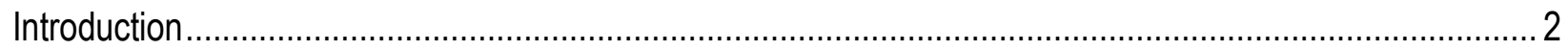

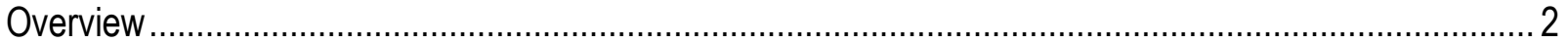

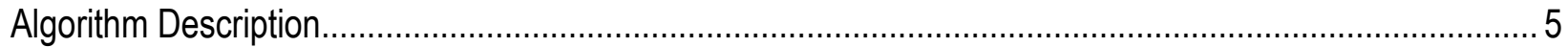

ASTER Level 1 input data.....................................................................................................

ASTER Level 1 Supplementary Algorithms ................................................................................. 5

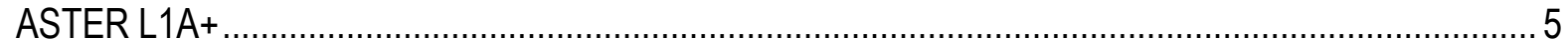

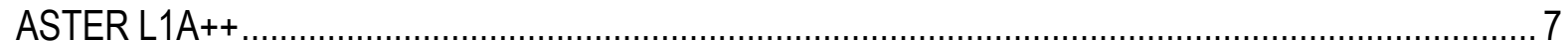

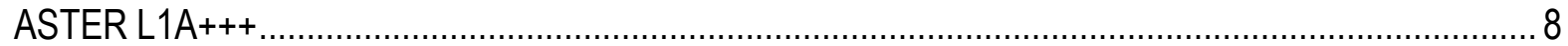

Cross-Talk Phenomenon Correction ........................................................................................... 11

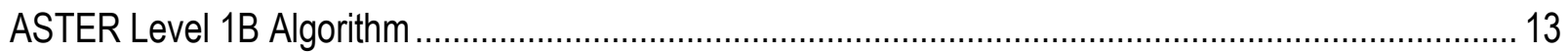

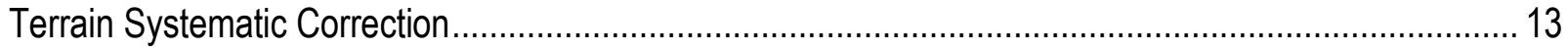

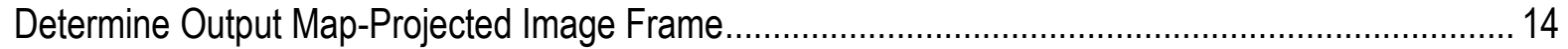

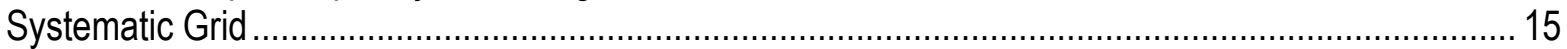

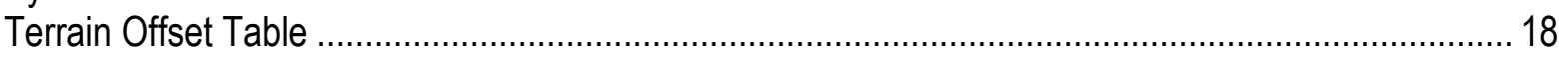

Resample DEM Using the Terrain Offset Grid........................................................................... 20

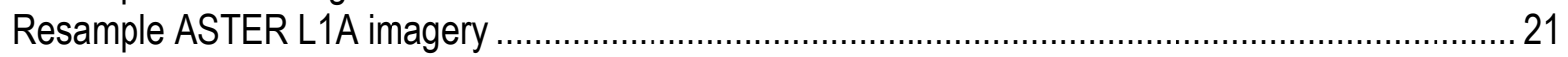

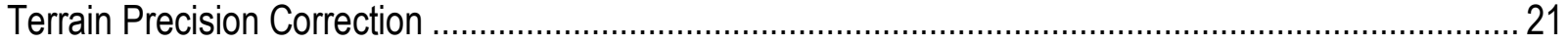

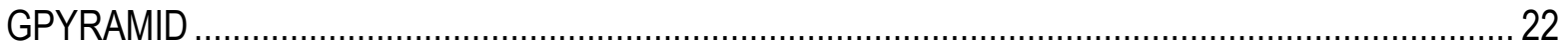

Correlate the Available Ground Control Points with the Systematic Image .....................................22

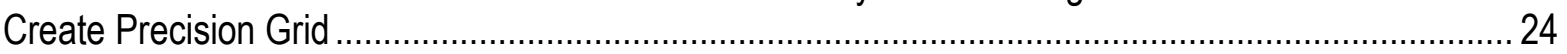

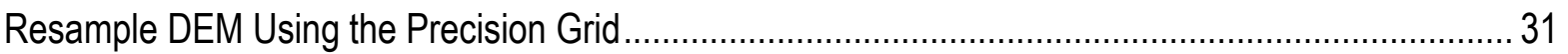

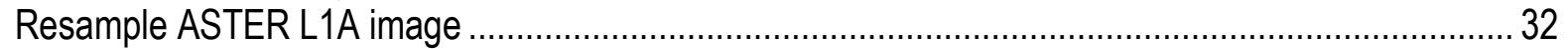

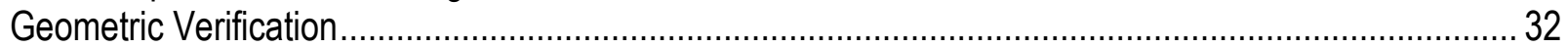

Full Resolution GeoTIFF Browse Images ............................................................................. 39

Advanced Spaceborne Thermal Emission and Reflection Radiometer Level 1 Precision Terrain Corrected

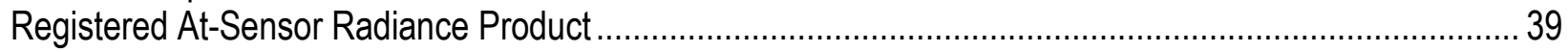

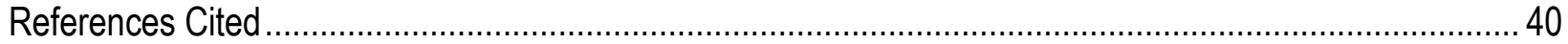

Appendix 1. ASTER Shortwave Infrared User Advisory July 18, 2008 ................................................ 42

Change in Status Alert - July 18, 2008 .................................................................................... 42

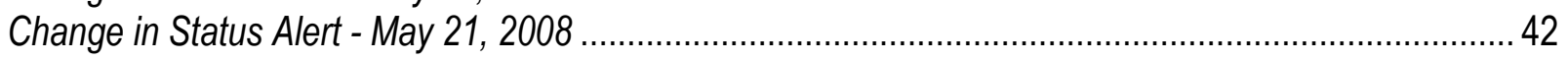

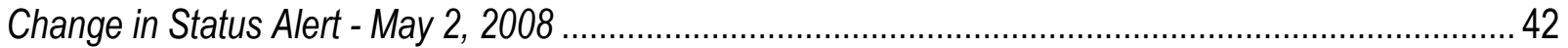

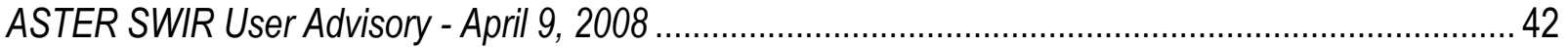

\section{Figures}

Figure 1. Overview of the AST_L1T algorithm.

Figure 2. Cross-track direction geolocation correction partitioned into latitude and longitude corrections as a function of scene orientation angle and scene center latitude (Land Processes Distributed Active Archive, 2012).

Figure 3. Visible Near Infrared on-board calibrator (used with permission from Arai and others, 2011b) ....8 Figure 4. RCC model trend graph $(A)$ shows band 1 , graph $(B)$ shows band 2 , and graph $(C)$ shows band 3 (K. Arai, written commun., 2014) 
Figure 5. ASTER L1A SWIR composites of Taraku Island, Japan (P. Scaramuzza, written commun., 2015). The image acquired April 28, 2001, at 01:22:41 UTC allowed for a good comparison to the figure published by Tonooka and Iwasaki in 2004.

Figure 6. Cross-talk mechanism originates from $A$ band 4 detectors and $B$ filter boundaries (A. Iwasaki, written commun., 2015).

Figure 7. SWIR Sensor Focal Plane Configuration (used with permission from Toonoka and Iwasaki, 2004)

Figure 8. Taraku Island band 5 after application of cross talk correction; image provided by Pat Scaramuzza?. 13

Figure 9. The bounding rectangle of an ASTER scene. 14

Figure 10. Simplistic example of image sample calculations. 15

Figure 11. ASTER Level 1 scene rotation with the amended ASTER Level 1 input coordinates as A, B, $C$, and $D$ on displayed on the left and the north up coordinates for $A, B, C$, and D displayed on the right. .15

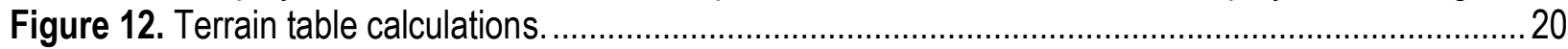

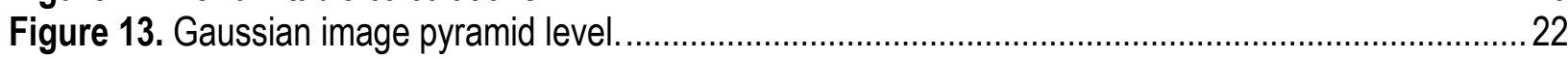

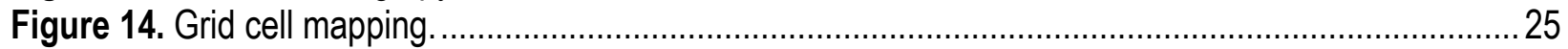

Figure 15. Curvature test for precision correction process................................................................. 31

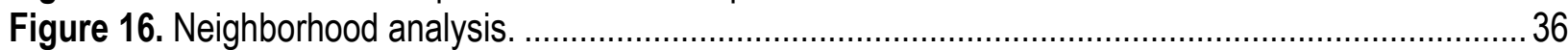

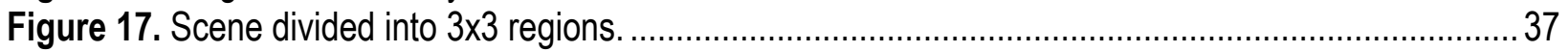

Figure 18. Example of browse image with valid GCP overlaid on an AST_L1T single band image............38

\section{Tables}

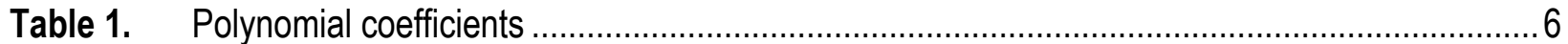

Table 2. Selected bands and pixel units of full resolution GeoTIFF browse images .............................39 


\begin{tabular}{ll} 
Abbreviations \\
ADD & Algorithm Description Document \\
AIST & National Institute of Advanced Industrial Science and Technology \\
ASTER & Advanced Spaceborne Thermal Emission and Reflection Radiometer \\
AST_L1T & ASTER Level 1 Precision Terrain Corrected Registered At-Sensor Radiance \\
ATBD & Algorithm Theoretical Basis Document \\
CCD & Charge-Coupled Device \\
DEM & Digital Elevation Model \\
DN & Digital Number \\
EROS & Earth Resources Observation and Science \\
ERSDAC & Earth Remote Sensing Data Analysis Center \\
EOS & Earth Observation System \\
ETM+ & Enhanced Thematic Mapper Plus \\
GCP & Ground Control Point \\
GCTP & General Cartographic Transformation Package \\
GDS & Ground Data System \\
GeoTIFF & Geographic Tagged Image File Format \\
GLS2000 & Global Land Survey 2000 \\
GSL & GNU Scientific Library \\
HDF & Hierarchical Data Format \\
LP DAAC & Land Processes Distributed Active Archive Center \\
LSfit & Least Squares Fit \\
LUT & Look-Up Table \\
MAD & Median Absolute Deviation \\
METI & Ministry of Economy, Trade and Industry \\
MMIO & Modified Moravec Interest Operator \\
MSS & Multispectral Scanner \\
NASA & National Aeronautics and Space Administration \\
OBC & On-board Calibrator \\
ODL & Object Definition Language \\
PGE & Product Generation Executable \\
PNG & Portable Network Graphics \\
QA & Quality Assurance \\
RMSE & Root Mean Square Error \\
S4PM & Simple, Scalable, Script-based Science Processor for Missions \\
SWIR & Shortwave Infrared \\
TIR & Thermal Infrared \\
USGS & U.S. Geological Survey \\
UTM & Universal Transverse Mercator \\
VNIR & Visible Near Infrared \\
WRS & Worldwide Reference System \\
ZMAD & Z-value Median Absolute Deviation \\
& \\
\hline
\end{tabular}




\title{
Advanced Spaceborne Thermal Emission and Reflection Radiometer Level 1 Precision Terrain Corrected Registered At-Sensor Radiance Product (AST_L1T), Algorithm Theoretical Basis Document
}

By David Meyer ${ }^{1}$, Dawn Siemonsma², Barbara Brooks ${ }^{3}$ and Lowell Johnson ${ }^{3}$

\author{
Abstract \\ This document provides an overview of the Advanced Spaceborne Thermal Emission and \\ Reflection Radiometer (ASTER) supplemental algorithms in conjunction with the reuse of \\ Landsat geometric algorithms modified by the National Aeronautics and Space Administration \\ (NASA Land Processes Distributed Active Archive Center (LP DAAC) to create an ASTER \\ Level 1 Precision Terrain Corrected Registered At-Sensor Radiance (AST_L1T) product. \\ Implementation of these algorithms occurs within the AST_L1T product generation executable \\ (PGE) as part of the open source Simple, Scalable, Script-based Science Processor for Missions \\ (S4PM) processing software subsystem. \\ The AST_L1T algorithms include the following: \\ - Generation of the AST_L1A input product via supplemental algorithms \\ - Application of the radiometric and geometric corrections appended in the AST_L1A \\ product \\ - Application of cross-talk correction coefficients \\ - Generation and application of affine transformation coefficients \\ - Modification and reuse of Landsat's geometric algorithms including: \\ - Systematic-generates the systematic grid \\ - Resampling - multi-use; only a single resample of input scene \\ - Gpyramid - scale input image to reference image (if necessary) \\ - Ground Control Point (GCP) Correlate - computes x,y offsets for GCPs to be used \\ for precision grid generation \\ - Precision Refine-generates the precision grid \\ - GVERIFY - geometric verification
}

\footnotetext{
${ }^{1}$ U.S. Geological Survey

2 Innovate! Inc., Contractor to the U.S. Geological Survey

${ }^{3}$ SGT Inc., Contractor to the U.S. Geological Survey
} 


\section{Introduction}

The ASTER Level 1 Precision Terrain Corrected Registered At-Sensor Radiance (AST_L1T) product originated at the NASA Land Processes Distributed Active Archive Center (LP DAAC) reuses the proven Landsat geometric algorithms with modifications for application to the ASTER dataset. The AST_L1T uses the appended radiometric and geometric corrections in the AST_L1A product and cross-talk corrections as the systematic basis on which to apply the terrain and precision corrections. The terrain and precision correction algorithms use Earth ellipsoid and terrain surface information (Global Land Survey 2000 Digital Elevation Model (GLS2000 DEM), derived ground control points), in conjunction with spacecraft ephemeris and attitude data, ASTER instrument off-nadir angle, and knowledge of the ASTER instrument and Terra satellite geometry to relate locations in the AST_L1A image path oriented perspective, geocentric space to geodetic object space (latitude, longitude, and height) in north up Universal Transverse Mercator (UTM) coordinate system. These algorithms create accurate AST_L1T output products characterizing the ASTER absolute and relative geometric accuracy. The AST_L1T product provides GIS-ready data with a single resampling from the AST_L1A.

This document compiles and synthesizes the applicable content from the following parent documents:ASTER Algorithm Theoretical Basis Document for ASTER Level-1 Data Processing Version 3 (Earth Remote Sensing Data Analysis Center, 1996), and the Multispectral Scanner (MSS) Geometric Algorithm Description Document (ADD) (U.S. Geological Survey, 2012).

\section{Overview}

Advanced Spaceborne Thermal Emission and Reflection Radiometer (ASTER) is a joint mission between the National Aeronautics and Space Administration (NASA) and Japan's Ministry of Economy, Trade and Industry (METI). The ASTER instrument aboard NASA's Terra satellite has a multitelescope configuration with 14 bands among 3 different sensor subsystems that include the following: Visible Near-Infrared (VNIR) 15 meter bands 1-3, Shortwave Infrared (SWIR) 30 meter bands 4-9, and Thermal Infrared (TIR) 90 meter bands 1014. The VNIR sensor has a cross-track pointing capability of plus or minus $24^{\circ}$ (degrees) offnadir, whereas the SWIR and TIR sensors are pointable at plus or minus $8.55^{\circ}$ off-nadir also in the cross-track direction. The ASTER instrument does not collect data continuously; instead, all observations are driven by data acquisition requests (DARs) submitted by the land science community.

The AST_L1T product produced by the LP DAAC consists mainly of two different levels of potential correction because of the variation in parameters among the AST_L1A input scenes. The two levels of correction are (1) terrain precision correction, applicable to all daytime AST_L1A scenes where correlation statistics reach a minimum threshold, and (2) terrain systematic correction, applied to all AST_L1A input data for which the precision correction is not possible, usually because of poor ground imaging that is, heavily clouded or night scenes containing only TIR bands, or where ground control is not available.

In addition to the two primary levels of correction identified in the previous paragraph, the AST_L1T software also allows for the absence of available terrain for correction, with two alternate levels: precision correction and systematic correction. These additional levels of correction follow the respective processes of the primary levels, except the elevation-related components are skipped. 
A high-level overview of the end-to-end processing flow for the AST_L1T product generation is provided in figure 1. The generation of the AST_L1A input scene appears at the top-most level of the flow chart (fig. 1), along with cross-talk correction, then the AST_L1B code which applies the inter- and intra- telescope registration correction, radiometric coefficients, and the affine transformation coefficients that are calculated and appended to the AST_L1A during product generation. At this point, the search image is determined. Because ASTER SWIR band 4 has a similar spatial and spectral resolution to Landsat's band 5, it is the preferred option for use in the modified Landsat geometric algorithm; however, if this band is not available, or the scene was acquired after April 2008 when SWIR data became saturated, then VNIR band 2 is used. Please refer to appendix 1 for more information regarding the SWIR anomaly.

Use of the modified Landsat geometric algorithm begins with the creation of the systematic grid where the amended AST_L1A input scene is rotated from the satellite path orientation to UTM north up orientation. The points in the rotated image are then mapped back to those of the initial AST_L1A input image space.

GLS2000 digital elevation model (DEM) tiles are retrieved and mosaicked to cover the systematic scene region. At this point, the algorithm must determine the level of correction that may be achieved.

- If the scene contains TIR bands only, then the DEM is resampled to match the systematic reference image, creating a matching pixel-for-pixel terrain dataset. The AST_L1A input scene is then resampled using the systematic grid and the terrain dataset. This resampling applies to all image bands.

- If the scene does not contain TIR bands only, then the algorithm passes the systematic grid and the intermediary terrain dataset to the terrain precision correction process, which begins with generating a precision grid. Creation of the precision grid is achieved by updating the systematic grid using ground control point (GCP) offsets computed by correlating the GLS2000 GCP image chips to points in the systematic band. This precision grid is then used to resample the terrain dataset. The next step is resampling the AST_L1A input scene (all bands) using the terrain dataset and the precision grid.

- When the input scene contains a large amount of cloud cover or other anomalies, the minimum correlations threshold needed to generate the precision grid may not be attainable. In these instances, the process will revert to the terrain systematic correction method.

This final resampling produces the ASTER Level 1 Precision Terrain Corrected Registered At-Sensor Radiance (AST_L1T) product. The AST_L1T product is GIS-ready data produced using a single resampling from the AST_L1A input scene. This multifile product includes 14 Hierarchical Data Format - Earth Observing System version 4 (HDF-EOS4) bands of ASTER L1T calibrated radiance data, full-resolution Geographic Tagged Image File Format (GeoTIFF) browse images of the VNIR-SWIR and TIR bands, low resolution thumbnail browse images, and an associated XML metadata file. 


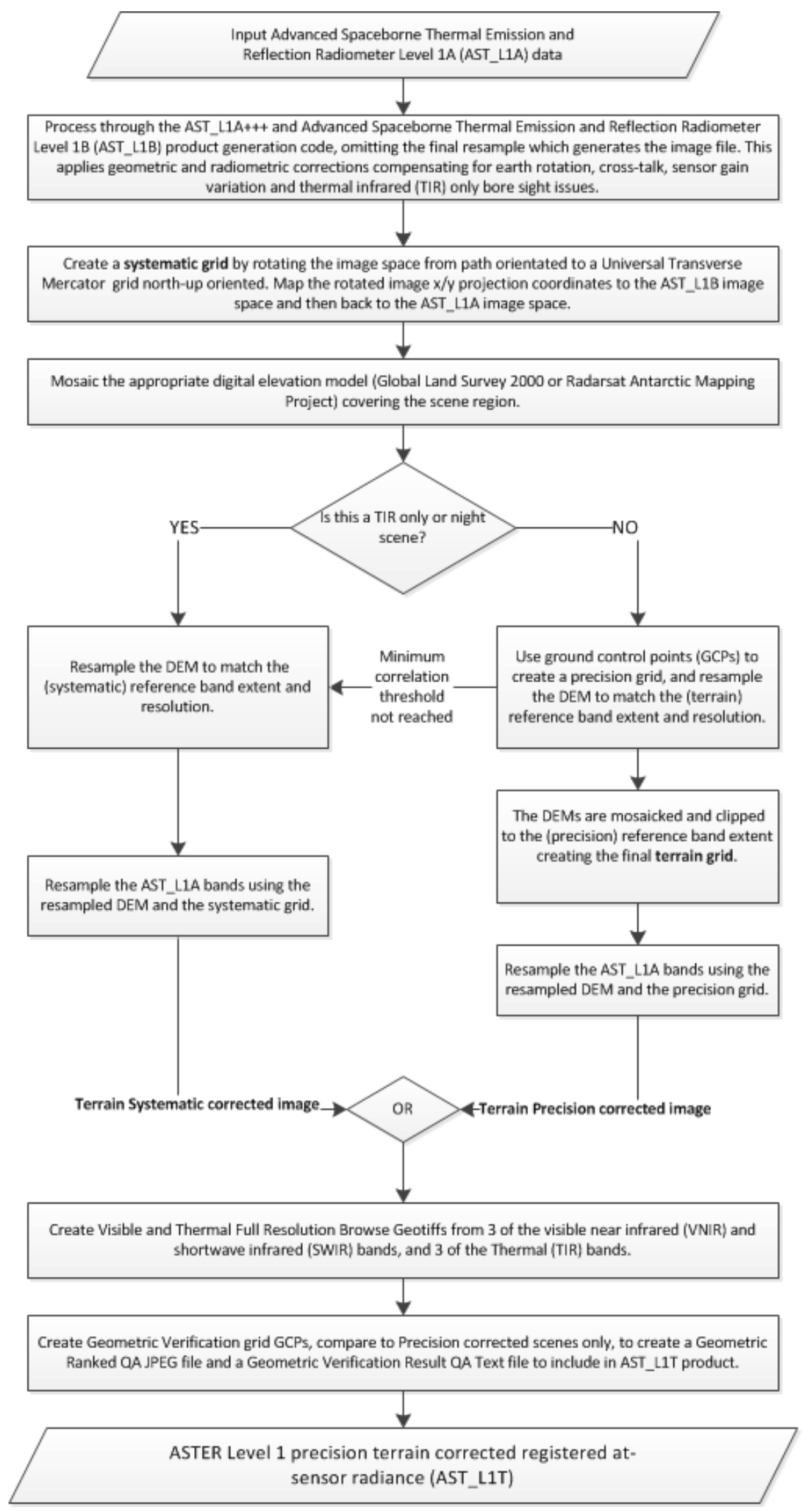

Figure 1. Overview of the AST_L1T algorithm. 


\section{Algorithm Description}

This section describes the various supplemental algorithms for implementing corrective modifications to the input AST_L1A product used in the generation of ASTER L1T. Next, the algorithms associated with the terrain systematic correction and terrain precision correction are described, followed by the subsequent sections describing the AST_L1T geometric verification and full resolution browse.

\section{ASTER Level 1 input data}

Japan Space Systems currently (2015) processes ASTER data from raw Level 0 instrument and satellite telemetry data to AST_L1A in the front-end processing module before electronically routing the data to the LP DAAC for archiving, subsequent processing, and distribution. The AST_L1A data product consists of image data, metadata, radiometric coefficients, geolocation data, and auxiliary data comprised of instrument supplement data and spacecraft ancillary data that are appended, but not applied. Please refer to the Algorithm Theoretical Basis Document for ASTER Level-1 Data Processing (Earth Remote Sensing Data Analysis Center, 1996) for additional information.

\section{ASTER Level 1 Supplementary Algorithms}

Beginning in 2006, AST_L1A data products are distributed on-demand. This change accommodated running the supplemental algorithms consecutively after the initial AST_L1A processing completed. The supplemental algorithms do not alter the original ASTER Level 1 algorithms; they are corrective in nature and address discrepancies that have affected the radiometric or geometric accuracy of the data over time. All AST_L1A data distributed to the science team and the public, in addition to L1A input provided for the generation of higher-level products, are processed with supplemental algorithms. Current (2015) supplemental algorithms include $\mathrm{L} 1 \mathrm{~A}+, \mathrm{L} 1 \mathrm{~A}++$, and $\mathrm{L} 1 \mathrm{~A}+++$.

\section{ASTER L1A+}

The AST_L1A+ supplemental algorithm was implemented on May 25, 2005, to address geolocation discrepancies caused by the following:

1. The Earth rotation angle, resulting from of an incorrect calculation of the Earth's rotation angle. This produced a geolocation error of as much as 300 meters near the poles for daytime scenes and less than 100 meters below 70 degrees latitude. The longitude error for nighttime scenes is largest at the equator, and decreases to approximately 100 meters at the poles. The sixth degree polynomial model provided in equation 1 along with the coefficients listed in table 1, and corrective locational calculations in equations 2 and 3 , can be used to express the approximate latitude and longitude errors for data produced before June 3, 2004, with geometric database versions older than 3.00

$$
\begin{gathered}
\Delta \lambda(\text { or } \Delta \varphi)=a_{0}+a_{2} \varphi_{C}^{2}+a_{4} \varphi_{C}^{4}+a_{6} \varphi_{C}^{6} \\
\lambda_{c}=\lambda_{m}-\Delta \lambda \\
\varphi_{c}=\varphi_{m}-\Delta \varphi
\end{gathered}
$$


where $\lambda \mathrm{c}$ is corrected longitude in degrees, $\lambda \mathrm{m}$ is measured longitude in degrees, $\Delta \lambda$ is longitude error in degrees, $\varphi c$ is corrected latitude in degrees, $\varphi m$ is measured latitude in degrees, and $\Delta \varphi$ is latitude error in degrees.

Table 1. Polynomial coefficients ${ }^{4}$

E signifies exponents of 10

\begin{tabular}{|c|c|c|c|c|}
\hline \multirow{2}{*}{ Coefficient } & \multicolumn{2}{|c|}{ Descending (daytime observation) } & \multicolumn{2}{c|}{ Ascending (night--time observation) } \\
\cline { 2 - 5 } & Longitude & Latitude & Longitude & Latitude \\
\hline$a_{0}$ & $-7.1636 \mathrm{E}-04$ & $1.2344 \mathrm{E}-04$ & $-7.0494 \mathrm{E}-03$ & $-9.6332 \mathrm{E}-04$ \\
\hline$a_{2}$ & $6.0217 \mathrm{E}-07$ & $-1.7743 \mathrm{E}-07$ & $4.0453 \mathrm{E}-07$ & $-1.7053 \mathrm{E}-07$ \\
\hline$a_{4}$ & $-4.9155 \mathrm{E}-11$ & $6.4363 \mathrm{E}-11$ & $-5.0296 \mathrm{E}-10$ & $5.9668 \mathrm{E}-11$ \\
\hline$a_{6}$ & $3.4510 \mathrm{E}-14$ & $-1.3692 \mathrm{E}-14$ & $4.8045 \mathrm{E}-14$ & $-1.2155 \mathrm{E}-14$ \\
\hline
\end{tabular}

2. The Earth nutation-related longitudinal error, which is affected by an omission of compensation for nutation, a slightly irregular oscillatory movement or wobbles in the axis of the Earth's rotation. The longitude error is dependent on the date of ASTER data acquisition. In general, the magnitude of error before July 2003 was less than 50 meters, but it increased to approximately 188 meters by the end of 2004, and grew to approximately 262 meters by April 2005. All ASTER Level 1 data distributed after May 2005 were produced with the ASTER L1A+ supplemental algorithm incorporating geometric database version 3.0 or later, which reduces this nutation error. Data produced between September 2003 and April 2005 can be corrected by post processing the data using equation 4 to calculate the longitudinal error (Earth Remote Sensing Data Analysis Center, 2005),

$$
\begin{aligned}
& \Delta \lambda_{n}=-4.386 * 10^{-3} \sin (\Omega)-3.36 * 10^{-4} \sin (L)-5.8 * 10^{-5} \sin (m)+5.3 * \\
& 10^{-5} \sin (2 \Omega)+3.6 * 10^{-5} \sin (S)+4.125 * 10^{-3} \quad(\text { deg })
\end{aligned}
$$

provided

$$
\begin{array}{lll}
\Omega=125.04-1934.14 T & (\mathrm{deg}) & \leftarrow 18.6 \text { years. } \\
L=560.93+72001.54 T & (\mathrm{deg}) & \leftarrow 182.6 \text { days } \\
m=436.63+962535.76 T & (\mathrm{deg}) & \leftarrow 13.7 \text { days } \\
S=357.53+35999.05 T & (\mathrm{deg}) & \leftarrow 1 \text { year }
\end{array}
$$

provided, $T$ is a Julian century beginning at noon January 1, 2000, as shown in equation 5.

$$
\mathrm{T}=(\mathrm{JD}-2451545) / 36525
$$

Julian date $J D$ can be calculated directly from Christian year (at noon in Gregorian calendar) by using the $T$ function, but it can also be obtained with equation 6 ,

$\mathrm{T}] \mathrm{D}=[365.25 \mathrm{Y}]+[\mathrm{Y} / 400]-[\mathrm{Y} / 100]+[30.59(\mathrm{M}-2)]+\mathrm{D}+1721088.5$

\footnotetext{
${ }^{4}$ Polynomial coefficients obtained from the Earth Remote Sensing Data Analysis Center, 2007.
} 
where the Christian year is in $Y$, month is in $M$, day is in $D$. However, $M$ for January is to be 13 and $M$ for February is to be 14 and the year $Y$ is to be $Y-1$ for both months. The brackets [ ] indicate that only the integer portion is used.

By subtracting the error in longitude, $\Delta \theta n$, from the longitude given in the position information of the ASTER product, the longitudinal error would be corrected. The error in terms of distance $\Delta X$ in meters follows in equation 7 ,

$$
\Delta X=6380 * 1000 * \cos (\varnothing) * \pi * \theta n / 180 \quad(m)
$$

provided that $\varnothing$ is the geocentric latitude of the target area, a rough calculation of the error in distance can be made.

\section{ASTER L1A++}

The AST_L1A++ supplemental algorithm implemented on May 9, 2012, uses geometric database version 3.02 or greater, to address geolocation discrepancies in the TIR bands for nighttime acquisitions of approximately 100-400 meters toward the cross-track direction. This crosstrack error contributes to latitude and longitude errors, because ASTER's orbit, in relation to geographic north, varies with latitude. Fitting a line, equation 8, to the cross-track direction error points produces a linear relation (Earth Remote Sensing Data Analysis Center, 2005)

$$
\Delta X=16^{*} \theta p t g+250 m
$$

where $\Delta \mathrm{X}$ is the cross-track correction, and $\boldsymbol{\theta}$ ptg is the pointing angle, which varies plus or minus 8.55 degrees. Applying simple geometric analysis yields partitioning of $\Delta \mathrm{X}$ into new latitude ( $\varphi \square$ new) and new longitude ( $\lambda \square$ new) values, as illustrated in figure 2 (Land Processes Distributed Active Archive, 2012).

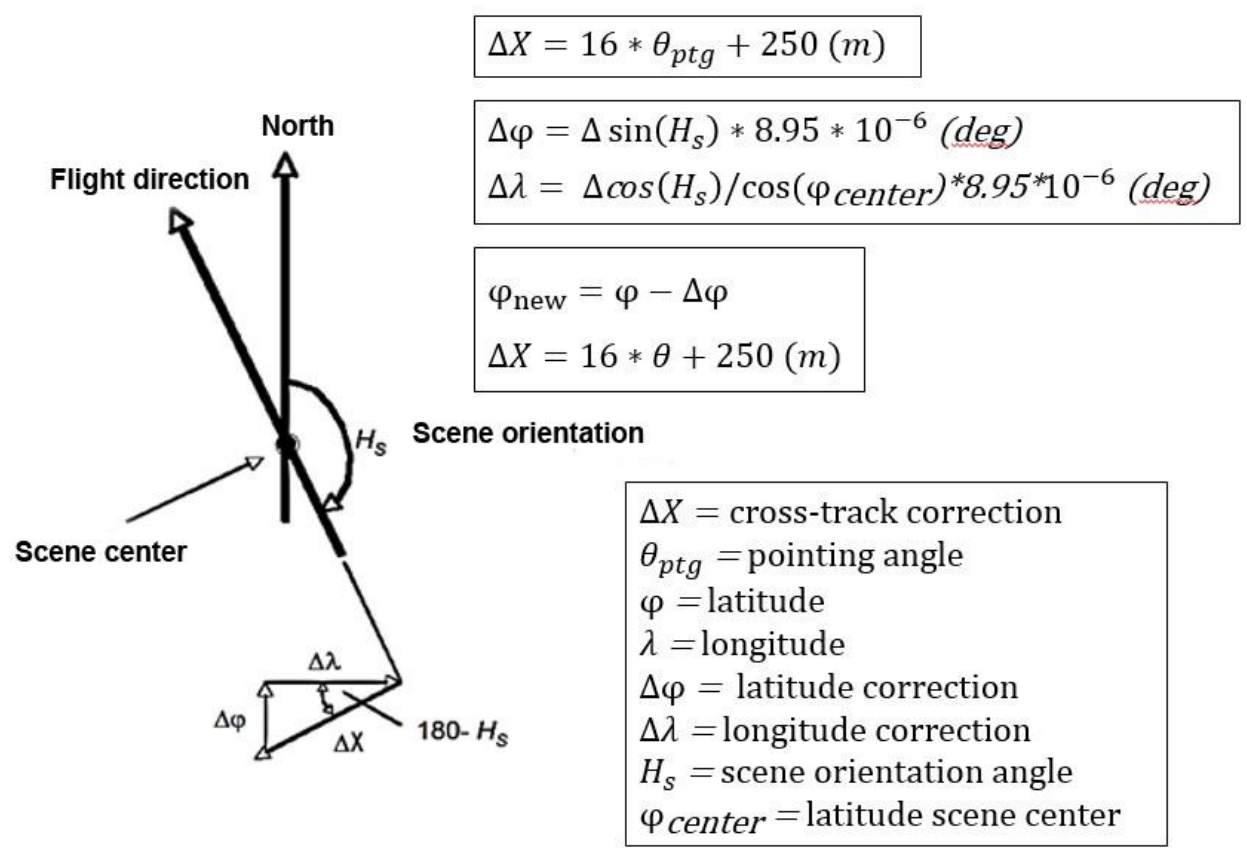

Figure 2. Cross-track direction geolocation correction partitioned into latitude and longitude corrections as a function of scene orientation angle and scene center latitude (Land Processes Distributed Active Archive, 2012). 


\section{ASTER L1A+++}

The ASTER L1A+++ supplemental algorithm implemented in late 2014 addresses degradation of the VNIR on-board calibrator (OBC) affected by dimming of the OBC halogen lamps over time.

The VNIR lamp-based calibration method, selected over alternate methods based on realestate limitations aboard the Terra platform, consists of two redundant onboard calibration halogen lamps (A and B) as shown in figure 3 (Arai and others, 2011b). Data collected from these lamps every 33 days (Arai and others, 2011a) are used to generate radiometric calibration coefficients (RCC) that are normalized using pre-flight data providing for a precise and repeatable means to monitor temporal trends in the radiometric response of the sensor.

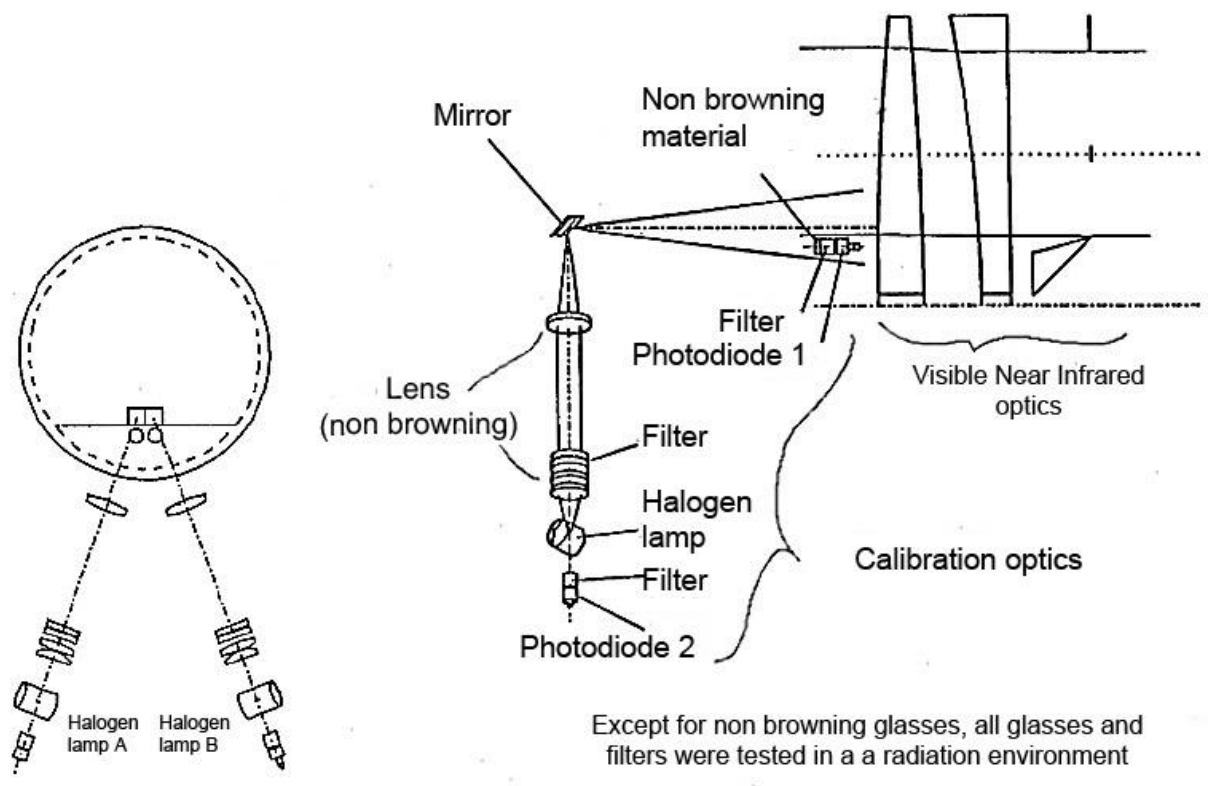

Figure 3. Visible Near Infrared on-board calibrator (used with permission from Arai and others, 2011b).

When the new RCC values deviate from the existing trend by 2 percent or more, the ASTER Science Team implements a new version of the RCC values (Thome and others, 2008) in the A processing to account for the uncertainty in the sensor response. Alternately, calibration may divert to cross-calibration inputs if the response from halogen lamps A and B are not consistent, and if all bands within a telescope do not display similar response tendencies, or to vicarious inputs if cross-calibration coefficients do not exist (Arai and others, 2011a).

Initially OBC RCC trends were determined using a linear function, however, since October 21, 2001, they have been approximated using an exponential function, equation 9, with a bias and a negative coefficient

$$
\text { function of RCC }=b * \exp (a * x)+c
$$

where $a$ is the offset term, $b$ is the slope/inclination, $c$ is the gain, and $x$ is the number of days since launch.

The ASTER VNIR OBC has been relatively stable; however, all spaceborne instruments gradually degrade over time. As a result, vicarious calibration conducted quarterly by the University of Arizona-United States, the National Institute of Advanced Industrial Science and 
Technology (AIST) — Japan, or the Saga University—Japan continues throughout the life of the mission.

Since launch, the average change in response is 23 percent for band 1,16 percent for band 2, and 10 percent for band 3 (Arai and others, 2011b). The RCC model trends for VNIR bands 1,2 , and 3 are shown in figure 4 . The thin orange line represents an overestimated trend of the degradation derived from the ASTER VNIR OBC radiometric model where the observed step changes occur on RCC update dates. The thick green line represents an average of vicarious calibrations for the VNIR bands with other instruments calibrated by AIST.

The AST_L1A+++ supplemental algorithm uses radiometric database version 4.13 constructed from the vicarious calibration/cross-calibration results (Arai, 2014) to account for the dimming of the $\mathrm{OBC}$ halogen lamps over time, because they more closely reflect the actual sensor degradation (D. Meyer, U.S. Geological Survey, written commun., 2014). 


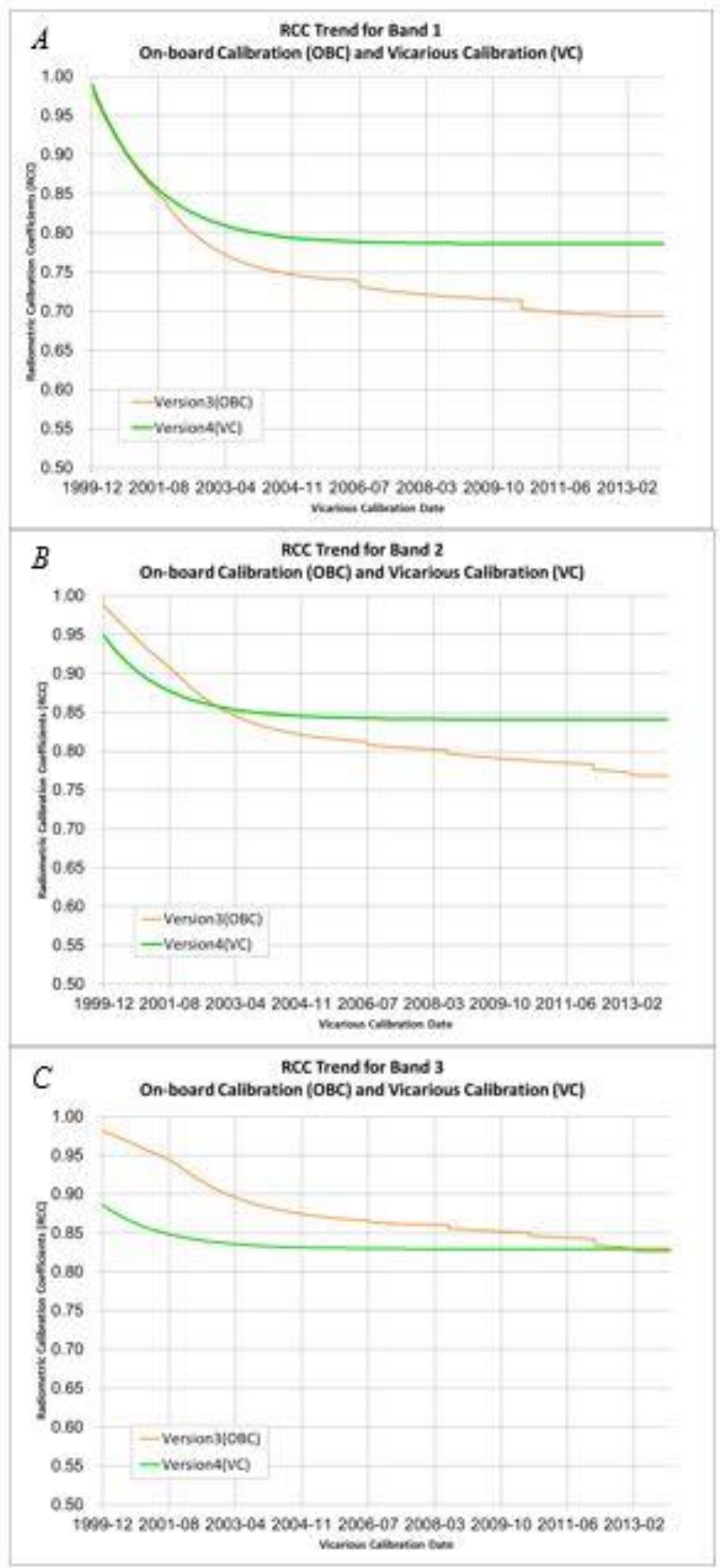

Figure 4. RCC model trend graph (A) shows band 1, graph (B) shows band 2, and graph (C) shows band 3 (K. Arai, written commun., 2014) 


\section{Cross-Talk Phenomenon Correction}

The cross-talk correction algorithm also is considered a supplementary algorithm, applied to the AST_L1A data for the ASTER L1T generation. Similar to the supplemental algorithms, discussed in the ASTER Level 1 Supplementary Algorithms section, the cross-talk correction algorithm does not modify the original ASTER Level 1 algorithms. The algorithm was developed to address the cross-talk phenomenon observed in SWIR bands 4-9 that are used for the detection of solar radiation reflected from the Earth's surface. Cross-talk is an optical leak from band 4 to the other SWIR bands resulting in a superimposed ghost image. This anomaly is shown for the along-track direction in figure 5 using images of bands $4-9$ without cross-talk correction (P. Scaramuzza, written commun., 2015). These images are a good comparison to the original figure published by Tonooka and Iwasaki in 2004, where the area simultaneously observed by bands 4-9 does not fall on exactly the same point on the ground. This anomaly occurs when band 4 incident light is reflected by the detector's aluminum-coated parts (especially from the area between the detector plane and band-pass filter), and is projected onto the other detectors as displayed in figure 6 (A. Iwasaki, written commun., 2015).
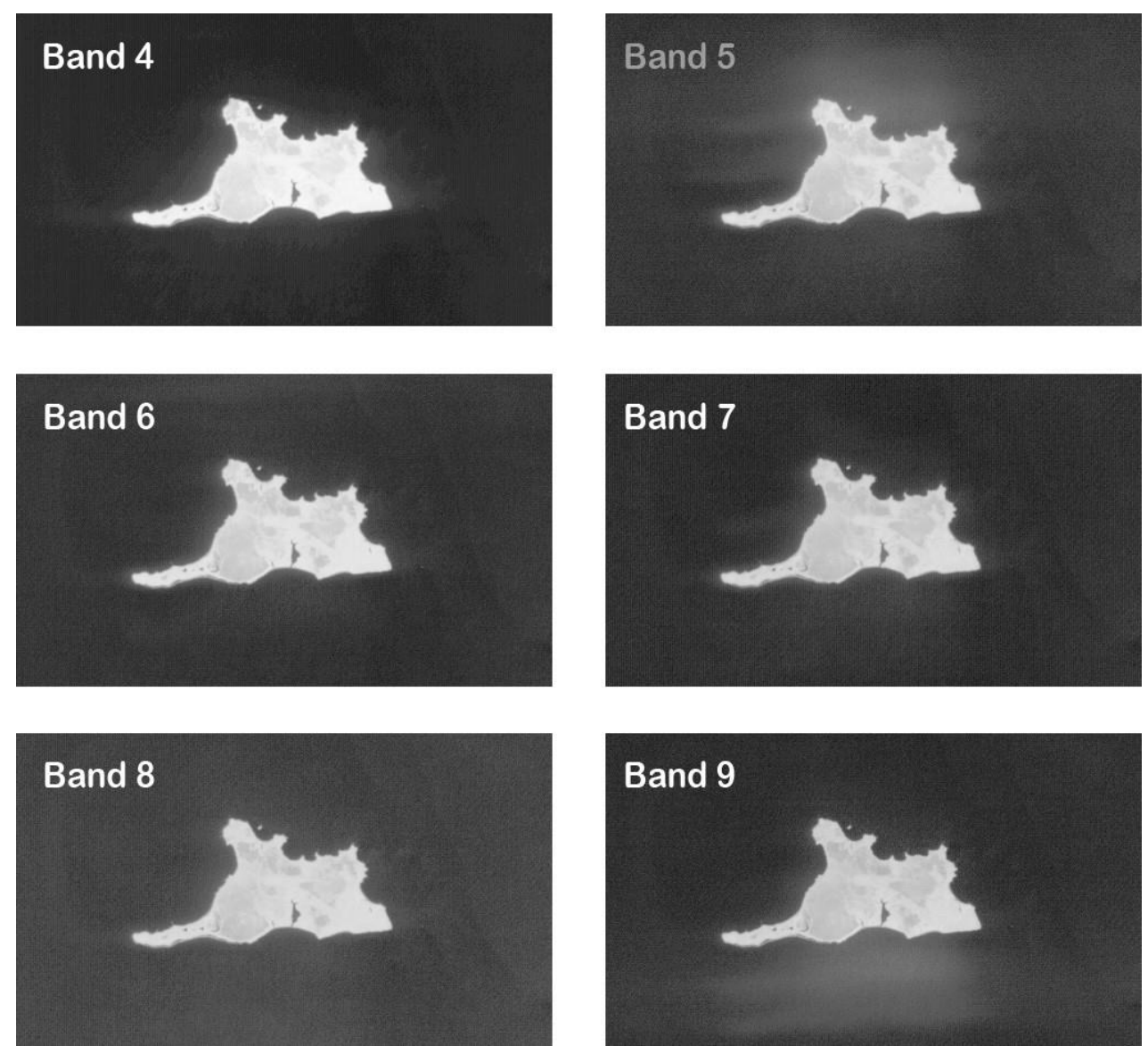

Figure 5. ASTER L1A SWIR composites of Taraku Island, Japan (P. Scaramuzza5, written commun., 2015). The image acquired April 28, 2001, at 01:22:41 UTC allowed for a good comparison to the figure published by Tonooka and Iwasaki in 2004.

${ }^{5}$ SGT Inc., Contractor to the U.S. Geological Survey 


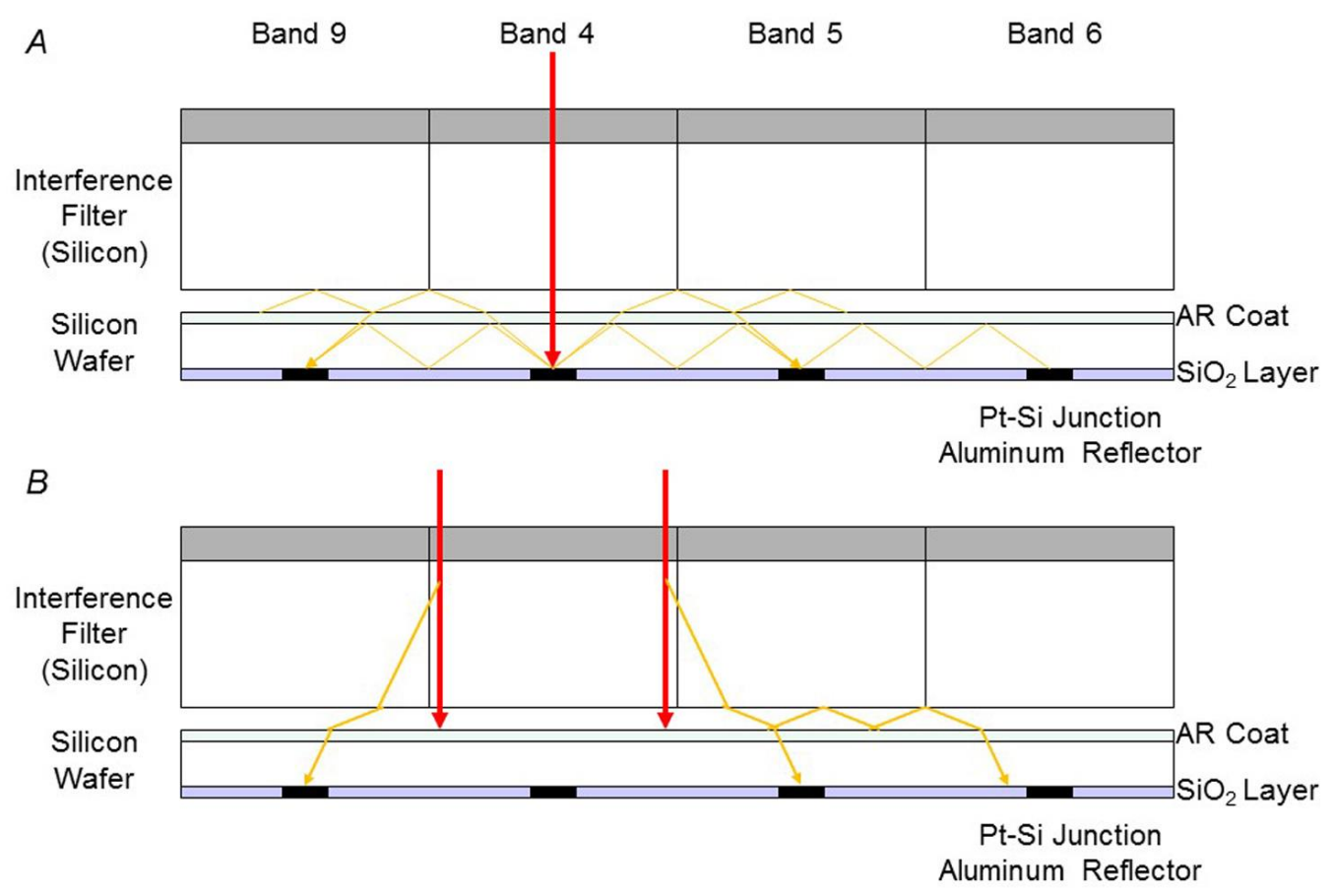

Figure 6. Cross-talk mechanism originates from A band 4 detectors and B filter boundaries (A. Iwasaki, written commun., 2015).

The cross-talk anomaly is further worsened by the band-to-band parallax effect and the distance between the charge-coupled device (CCD) array pairs shown in figure 7. Bands 9 and 5 display the most dominant parallax effects because of their locational proximity to the band 4 detectors. The spectral range of band 4 is between 1.6 and 1.7 micrometers $(\mu \mathrm{m})(0.092 \mu \mathrm{m}$ bandwidth), which is not only the widest bandwidth compared to the rest of the SWIR bands (average of $0.052 \mu \mathrm{m}$ bandwidth for bands 5 through 9), but is also the strongest in its reflectivity component. Therefore, the incident radiation of band 4 is about 4 to 5 times stronger than that of the other bands (B. Ramachandran, written commun., 2006).

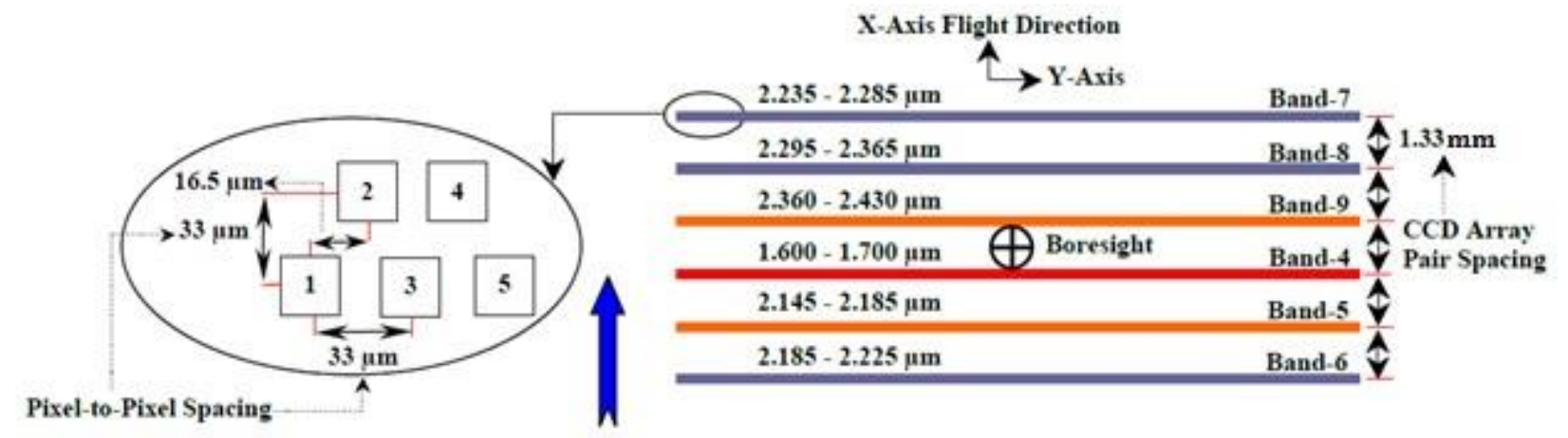

Figure 7. SWIR Sensor Focal Plane Configuration (used with permission from Toonoka and Iwasaki, 2004) 
The cross-talk corrected image of band 5 from Taraku Island, Japan (fig. 5), is shown in figure 8 .

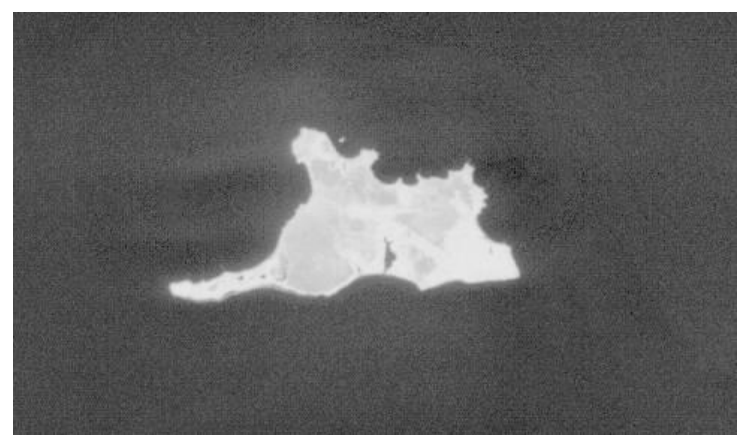

Figure 8. Taraku Island band 5 after application of cross talk correction; image provided by Pat Scaramuzza 5 .

\section{ASTER Level 1B Algorithm}

The ASTER Level 1B (AST_L1B) algorithm applies the radiometric calibration coefficients that are calculated and appended, but not applied to the distributed AST_L1A data product. These appended coefficients also include corrections for the DC Clamp phenomenon (Earth Remote Sensing Data Analysis Center, 2007), and inter- and intra-telescope bore alignment. Additionally, the algorithm transforms the path oriented coordinates to UTM coordinates, which are then linked back to the original AST_L1A input image coordinates using a set of eight pseudo-affine transformation coefficients per block, expressed by the pixel size units of each band.

Bad pixel values, for example, pixels included in missing packets during down link, damaged detectors, and all AST_L1B pixels generated from bad pixels in an input AST_L1A scene, are evaluated and corrected, using linear interpolation to generate replacement values when feasible (Earth Remote Sensing Data Analysis Center, 2007), before radiance is converted to Digital Number (DN) values taking into account normal gain and the gain factors included within the radiometric database. Please refer to the ASTER Algorithm Theoretical Basis Document for ASTER Level-1 Data Processing Version 3 (Earth Remote Sensing Data Analysis Center, 1996) for a more detailed description of ASTER L1B processing.

\section{Terrain Systematic Correction}

ASTER terrain systematic correction compensates for distortion in ASTER Level 1A data resulting from topographical variations and image data acquired with cross-track pointing angles that are off-nadir. ASTER terrain systematic correction includes determining the output map-projected image space, creating the systematic grid, mosaicking the GLS2000 digital elevation model data, clipping the GLS2000 mosaic to match the scene boundaries, and resampling the DEM to match the final ASTER L1T image space. The ASTER Level 1A input image is then resampled using the systematic grid and the matching DEM to create the terrain systematic corrected image, which is the default for nighttime-only scenes, scenes that contain a large amount of cloud cover, and scenes that fail to create the precision grid necessary for the terrain precision correction process. 


\section{Determine Output Map-Projected Image Frame}

The first step of the process is to determine the geographic extent of the output image by choosing the initial set of projection coordinates mapped to the ASTER Level 1 input space after applying the appended radiometric and affine transformation coefficients.

The following steps describe the algorithm:

1. Initialize the target coordinates.

2. Determine the bounding rectangle from the SceneFourCorners, displayed in figure 9.

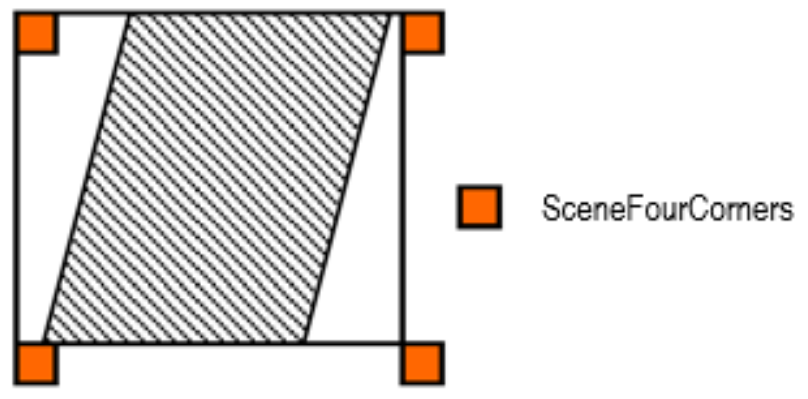

Figure 9. The bounding rectangle of an ASTER scene

3. Adjust for different pixel sizes among the bands. Since the scene framing can be arbitrarily larger than the image boundaries, ensure that the frame corners are multiples of the largest pixel size (in meters). This will also ensure that the corner coordinates are evenly divisible by all band resolutions. With the 90 meter TIR bands being the largest resolution, equations 10-13 show the minimum and maximum $x, y$ framing.

$$
\begin{aligned}
& x_{\min } \Rightarrow 90 *\left\lfloor x_{\min } / 90\right\rfloor \\
& x_{\max } \Longrightarrow 90 *\left\lceil x_{\max } / 90\right\rceil \\
& y_{\min } \Rightarrow 90 *\left\lfloor y_{\min } / 90\right\rfloor \\
& y_{\max } \Longrightarrow 90 *\left\lceil y_{\max } / 90\right\rceil
\end{aligned}
$$

(Note the floor and ceiling operators.)

The number of output image lines $\left(n_{l}\right)$ and samples $\left(n_{s}\right)$ for a given band are determined by dividing the $x$ and $y$ range by the pixel size $\left(p_{x}, p_{y}\right)$ as shown in equations 14 and 15 .

$$
\begin{aligned}
& n_{s}=\left(x_{\max }-x_{\text {min }}\right) / p_{x}+1 \\
& n_{l}=\left(y_{\max }-y_{\min }\right) / p_{y}+1
\end{aligned}
$$

The addition of 1 in equations 14 and 15 is needed because the frame bounds are centered on the boundary pixels. The graphic in figure 10 displays a simplified example of the sample calculations. 


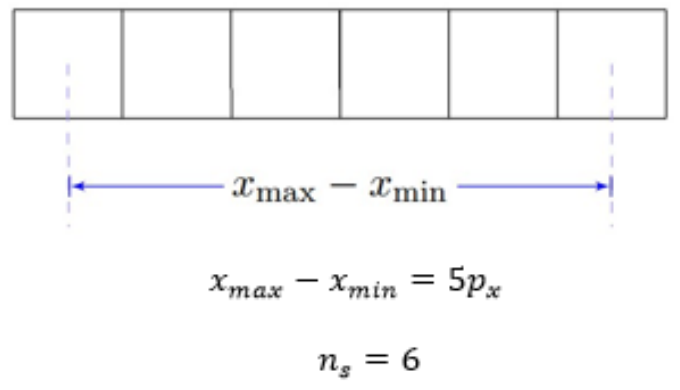

Figure 10. Simplistic example of image sample calculations.

\section{Systematic Grid}

The UTM north up systematic grid is generated by projecting the output points of the rotated image back to the ASTER Level 1A image space, as shown in figure 11. This is accomplished by using the rotation angle and the ASTER Level 1 image map to plot the output image space back to the generated ASTER Level 1 image space, and then to the input ASTER L1A space.

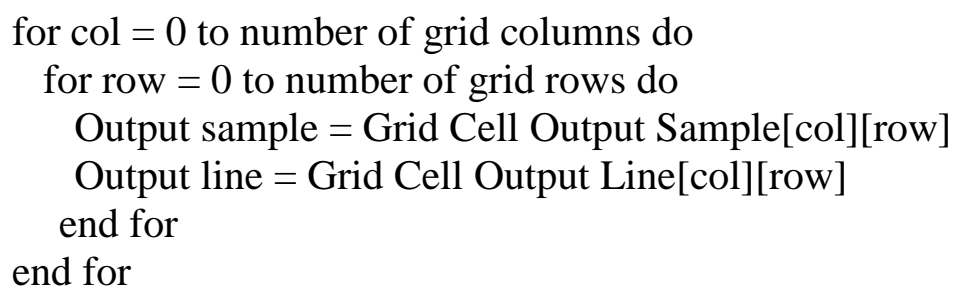

A

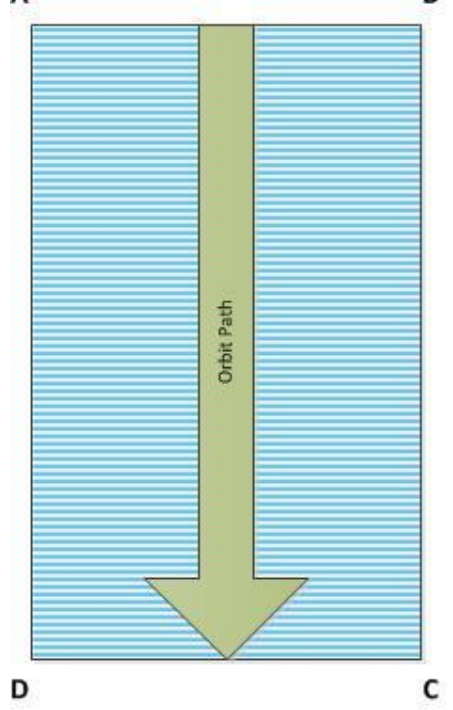

ASTER Level 1

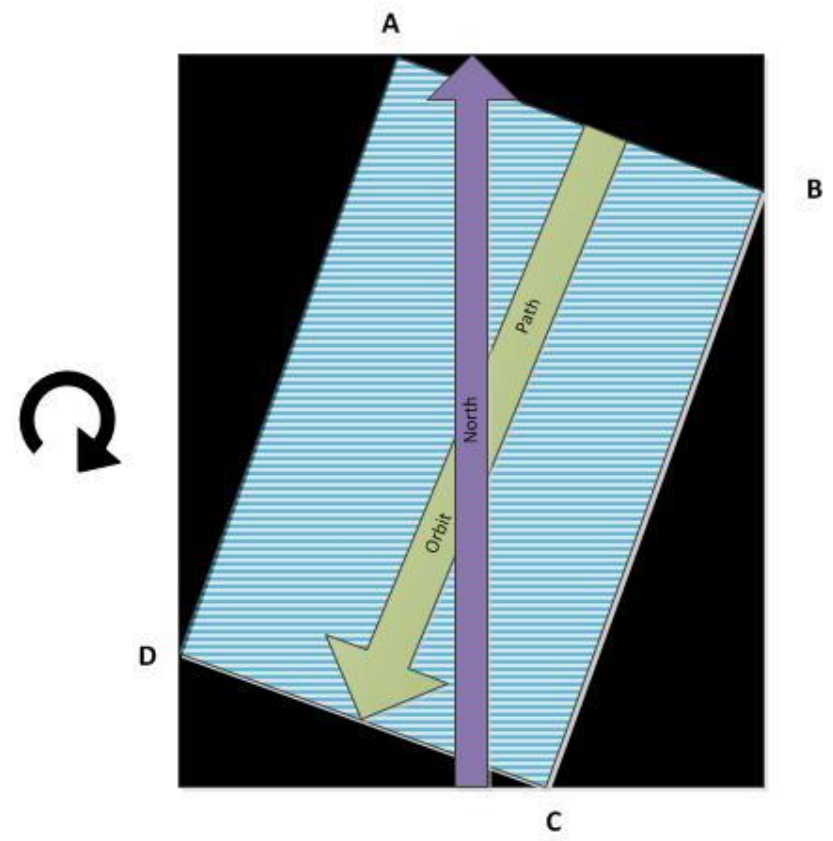

AST_L1T Scene

UTM Grid North-Up

Figure 11. ASTER Level 1 scene rotation with the amended ASTER Level 1 input coordinates as $A, B, C$, and $D$ on displayed on the left and the north up coordinates for $A, B, C$, and $D$ displayed on the right. 
1. Calculate the output projection coordinates using equations 16 and 17.

$$
\begin{gathered}
X_{u t}=\text { upper left targetx }+(\text { output sample }-1) \text { grid pixel sizex } \\
Y_{u t m}=\text { upper left targety }-(\text { output line }-1) \text { grid pixel sizey }
\end{gathered}
$$

2. Convert the map projection coordinates.

3. Convert the UTM map projection coordinates from meters to radians. The U.S. Geological Survey (USGS) General Cartographic Transformation Package (GCTP) converts from the UTM output projection to a geographic coordinate system (Xgeo, Ygeo). If Xgeo is less than 0, add $2 \pi$.

Determine individual grid cell mapping coefficients.

Bilinear mapping coefficients for each grid cell are calculated for mapping from input location to output location (forward mapping), and for mapping from output location to input location (reverse mapping). A separate mapping function is used for lines and samples, which equates to four mapping functions. A set of four mapping functions is calculated for each grid cell and for every band stored in the grid.

The following methodology calculates one set of four bilinear mapping equations: A 9 x 4 matrix fits nine points within a grid cell (4 corners, 4 edge midpoints, and cell midpoint). The matrix equation is shown in equation 18 ,

$$
[A][\text { coeff }]=[b]
$$

where matrix $A$ is $9 \times 4$, vector $b$ is $9 \times 1$, and the coefficient matrix, coeff, is $4 \times 1$. The coefficient matrix can be solved using equation 19 to obtain the mapping coefficients.

$$
[\text { coeff }]=\left[A^{T} A\right]^{-1}\left[A^{T} b\right]
$$

In the case of solving for an equation to map an input line and sample location to an output sample location belonging to one grid cell, the matrices can be defined as in equations $20-36$,

$$
A_{n, 0}=1
$$

where $n=0,8$

$$
\begin{gathered}
A_{4,1}=\left(A_{0,1}+A_{1,1}+A_{2,1}+A_{3,1}\right) / 4 \\
A_{5,1}=\left(A_{0,1}+A_{1,1}\right) / 2 \\
A_{6,1}=\left(A_{1,1}+A_{3,1}\right) / 2 \\
A_{7,1}=\left(A_{2,1}+A_{3,1}\right) / 2 \\
A_{8,1}=\left(A_{2,1}+A_{0,1}\right) / 2
\end{gathered}
$$

where $A_{0,1}$ is the upper left input sample location for current grid cell, $A_{1,1}$ is the upper right input sample location for current grid cell, $A_{2,1}$ is the lower left input sample location for current grid cell, and $A_{3,1}$ is the lower right input sample location for current grid cell

$$
\begin{gathered}
A_{4,2}=\left(A_{0,2}+A_{1,2}+A_{2,2}+A_{3,2}\right) / 4 \\
A_{5,2}=\left(A_{0,2}+A_{1,2}\right) / 2
\end{gathered}
$$




$$
\begin{aligned}
& A_{6,2}=\left(A_{1,2}+A_{3,2}\right) / 2 \\
& A_{7,2}=\left(A_{2,2}+A_{0,2}\right) / 2
\end{aligned}
$$

where $A_{0,2}$ is the upper left input line location for current grid cell, $A_{1,2}$ is the upper right input line location for current grid cell, $A_{2,2}$ is the lower left input line location for current grid cell, and $A_{3,2}$ is the lower right input line location for current grid cell

$$
\begin{gathered}
A_{8,2}=\left(A_{n, 1}+A_{n, 2}\right) / 2 \\
A_{n, 3}=A_{n, 1}, A_{n, 2}
\end{gathered}
$$

where $n=0,8$

$$
\begin{gathered}
b_{4}=\left(b_{0}+b_{1}+b_{2}+b_{3}\right) / 4 \\
b_{5}=\left(b_{0}+b_{1}\right) / 2 \\
b_{6}=\left(b_{1}+b_{2}\right) / 2 \\
b_{7}=\left(b_{2}+b_{3}\right) / 2 \\
b_{8}=\left(b_{2}+b_{0}\right) / 2
\end{gathered}
$$

where $b_{0}$ is the upper left output sample location for current grid cell, $b_{1}$ is the upper right output sample location for current grid cell, $b_{2}$ is the lower left output sample location for current grid cell, and $b_{3}$ is the lower right output sample location for current grid cell. The line and sample locations listed are defined at the grid cell corner coordinates. The points interpolated between the grid cell line segments provide stability for what could be, most notably, a mapping that involves a $45^{\circ}$ rotation, an ill-defined solution if only four points were used in the calculation. The set of coefficients define a bilinear mapping equation of the form presented in equation 37 ,

$$
\text { sample }_{o}=\operatorname{coeff}_{o}+\operatorname{coeff}_{1} * \operatorname{sample}_{\mathrm{i}}+\operatorname{coeff}_{2} * \text { linei }+\operatorname{coeff}_{3} * \text { sample }_{\mathrm{i}} *
$$
line $_{i}$

where sampleo is output sample location, samplei is input sample location, and linei is input line location.

A set of sample and line coefficients, equations 38-39, map the output projection space to the ASTER Level 1 space, and a set of sample and line coefficients, equations 40-41, map the ASTER Level 1 space to the output projection sample. line $_{i}$

sample $_{\mathrm{o}}=\operatorname{coeff}_{0}+\operatorname{coeff}_{1} * \operatorname{sample}_{\mathrm{i}}+\operatorname{coeff}_{2} * \operatorname{line}_{\mathrm{i}}+\operatorname{coeff}_{3} * \operatorname{sample}_{\mathrm{i}} *$

$$
\begin{aligned}
& \text { line }_{o}=\operatorname{coeff}_{4}+\operatorname{coeff}_{5} * \text { sample }_{i}+\operatorname{coeff}_{6} * \operatorname{line}_{\mathrm{i}}+\operatorname{coeff}_{7} * \text { sample }_{\mathrm{i}} * \text { line }_{\mathrm{i}} \\
& \text { sample }_{\mathrm{i}}=\operatorname{coeff}_{8}+\operatorname{coeff}_{9} * \text { sample }_{\mathrm{o}}+\operatorname{coeff}_{10} * \text { line }_{o}+\operatorname{coeff}_{11} * \text { sample }_{o} * \\
& \text { line }_{o} \\
& \text { line }_{i}=\operatorname{coeff}_{12}+\operatorname{coeff}_{13} * \text { sample }_{\mathrm{o}}+\operatorname{coeff}_{14} * \text { line }_{\mathrm{o}}+\operatorname{coeff}_{15} * \text { sample }_{\mathrm{o}} * \\
& \text { line }_{\mathrm{o}}
\end{aligned}
$$

The forward mapping equations, mapping input line, and sample locations to output line locations can be solved by swapping output line locations for output sample locations in the matrix $[b]$. The reverse mapping equations, mapping output locations to input line 
and sample, can be found using output line and sample locations in the $[A]$ matrix and the corresponding input sample and line locations in the $[b]$ matrix.

4. Calculate inverse mapping coefficients.

5. Calculate forward mapping coefficients.

6. Calculate the rough transform polynomial.

Calculate the rough mapping coefficients for the grid. These define a set of polynomials that map output line and sample locations to input line and sample locations. The rough polynomial is generated using a large number of points distributed over the entire scene to solve, a polynomial equation that maps an output location to an input location. The rough polynomial is only meant to get a "close" approximation of the input line and sample location for a corresponding output line and sample location. Once this approximation is made, the value can be refined for a more accurate solution. A rough mapping polynomial is found for every band.

7. A bilinear polynomial is used for rough mapping. The mapping function is therefore similar to functions used for each individual grid cell; however, the setup of the matrice, shown in equation 42 , to solve for the mapping coefficients are different,

$$
[A][\text { coeff }]=[b]
$$

where matrix $[A]$ is $\mathrm{N} \times 4$ and defined by the output line and sample locations, the coefficient matrix is $4 \times 1$, matrix $[b]$ is $\mathrm{N} \times 1$ and defined by either the input lines or input samples, and $N$ is equal to the total number of points stored in the grid for one image band. The rough polynomial is therefore determined by using all the point locations stored in the grid for a given band. One mapping is available for output line and sample location to input sample location, and one mapping is available for output line and sample location to input line location.

\section{Terrain Offset Table}

Terrain or relief effects are addressed by adjusting the sample location produced with the systematic grid, generated in the previous step, based on the off-nadir across- and along-track viewing angles and elevation height associated with the output projection coordinate of the ASTER Level 1 pixel.

The GLS2000 digital elevation model data is mosaicked and clipped to match the scene boundaries of the systematic image. To avoid performing the calculations for each output pixel, a look-up table (LUT) based on the off-nadir viewing angles and height, or elevation, is created. For any given elevation and angle associated with the ASTER L1T data, a delta pixel adjusts the sample location of the ASTER Level 1.

Steps to build the terrain look-up table follow:

1. Determine the bounds of terrain table parameters.

A. Given a nominal satellite orbital radius, set this value as the magnitude of the satellite positional vector.

B. Read DEM and determine the minimum and maximum elevation values, equation 43,

$$
\text { number of elevation values }=\frac{\text { maximum elevation-minimum elevation }}{\text { elevation increment }}+1
$$


where elevation increment is set to 1 meter. Build the nadir sample location by determining the nadir location using equations 44-48

$$
\begin{gathered}
\mathrm{sca}=\arcsin \left(\frac{R_{0}}{R_{e}} * \sin (p a)\right)-p a \\
p a_{a d j}=R_{e} * \frac{s c}{\text { pixsize }}
\end{gathered}
$$

$$
\text { maximum nadir }=\frac{1}{2} * \text { number of samples in ASTER L } 1+p a_{a d j}
$$

for $\mathrm{i}=0$ to number of lines in ASTER Level 1 do

$$
\operatorname{nadir}_{i}=\frac{1}{2}\left(\text { number of samples in ASTER Level1 }+p a_{a d j}\right)
$$

end for

$$
\text { number off }- \text { nadir samples }=\text { maximum nadir }
$$

where $p a$ is the pointing angle in radians, $R_{e}$ is the radius of the earth in meters, $R_{0}$ is the radius of the orbit in meters, sca is the scene-center earth angle, and pixsize is the pixel size in meters.

C. Calculate the terrain LUT. See figure 12 and equations 49-57.

for sample $=0$ to number of off-nadir samples do

for elev=minimum elevation to maximum elevation do

$$
g_{r}=(\text { pixel size } * \text { sample })
$$

where $g_{r}$ is the ground range in meters

$$
s=g_{r} / R_{e}
$$

where $s$ is the sample center earth angle from nadir in radians

$$
\operatorname{los}=\sqrt{R_{e}^{2}+\left(R_{e}+\text { alt }\right)^{2}-2 R_{e}\left(R_{e}+\text { alt }\right) \cos s}
$$

where los is the magnitude of the line of sight (LOS) vector in meters and alt is the altitude of the sensor platform in meters

$$
\beta=\sin ^{-1}\left(\frac{R_{e}}{l o s} \sin s\right)
$$

where $\beta$ is the instantaneous field of view (IFOV) from nadir to the LOS vector in radians

$$
d \theta=\tan ^{-1}\left(\frac{e l e v R_{0} \sin \beta / R_{e}}{\left(R_{e}+a l t\right) \cos \beta-\left(R_{e}+e l e v\right) \sqrt{1-\left(R_{e}+a l t\right)^{2} \frac{\sin ^{2} \beta}{R_{e}^{2}}}}\right)
$$

where $d \theta$ is the IFOV at the LOS vector as a function of elevation in radians

$$
Z p p=\sin -1\left(\left(R_{e}+a l t\right) \sin \left((\beta+d \theta) / R_{e}\right)\right)
$$

where $Z p p$ is the exterior angle for the IFOV angles as a function of $\beta$ and $d \theta$ in radians 


$$
d s=z_{p p}-s-\beta-d \theta
$$

where $d s$ is the sample center earth angle for current sample in radians

$$
d g_{r}=R_{e} d s
$$

where $d g_{r}$ is the arc length for the current sample and elevation in meters

$$
\text { terrain offset }[\text { sample }][\text { elev }]=\frac{d g_{r}}{\text { pixel size }}
$$

where terrain offset is the table of offsets in pixels

end for

end for

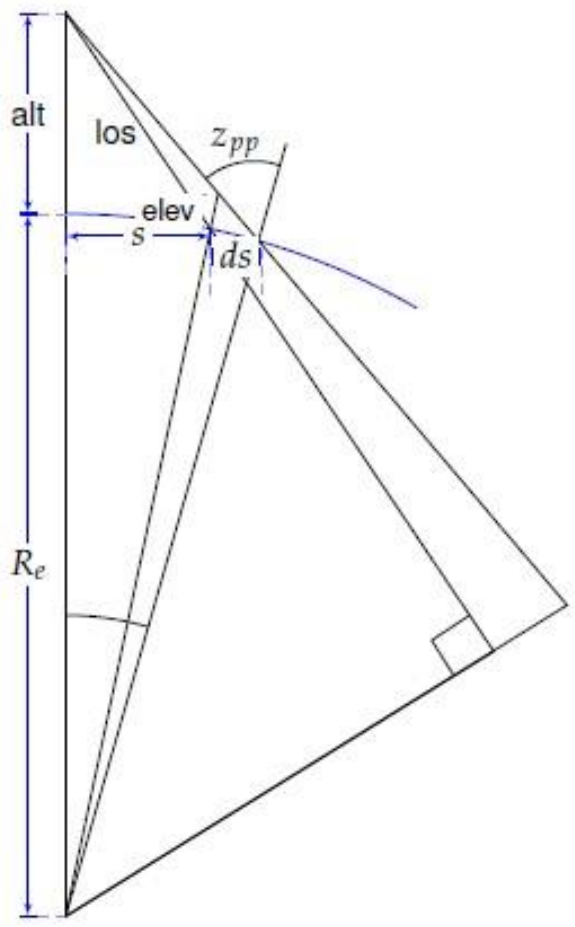

Figure 12. Terrain table calculations.

\section{Resample DEM Using the Terrain Offset Grid} image.

In this step, the DEM image is resampled using the terrain grid to match the systematic

for line $=0$ to number of output lines do

for sample $=0$ to number of output samples do

if producing AST_L1T then

Extract the corresponding elevation from the DEM.

Map the AST_L1T line/sample to the corresponding (path oriented) ASTER Level 1 line/sample using the grid.

Use the elevation and the ASTER Level 1 line sample to look up the sample offset from the terrain table.

Interpolate the output pixel value at the calculated Level 1A coordinates. 
end if

Determine grid cell number for output pixel location using equations 58-60.

$$
\begin{gathered}
\text { row }=\frac{\text { oline }}{\text { number }} \text { of grid rows } \\
\text { column }=\frac{\text { osamp }}{\text { number }} \text { of grid columns } \\
\text { grid cell index }=\text { row } * \text { number grid columns }+ \text { column }
\end{gathered}
$$

where oline is offset line and osamp is offset sample.

Read the grid cell inverse mapping coefficients for the grid cell number.

Calculate the input line and sample location using equations 61-63.

$$
\begin{gathered}
\text { sample }_{i}=\operatorname{coeff}_{8}+\operatorname{coeff}_{9} * \text { sample }_{\mathrm{o}}+\operatorname{coeff}_{10} * \operatorname{line}_{\mathrm{o}}+\operatorname{coeff}_{11} * \text { sample }_{\mathrm{o}} * \operatorname{line}_{\mathrm{o}} \\
\text { line }_{\mathrm{i}}=\operatorname{coeff}_{12}+\operatorname{coeff}_{13} * \operatorname{sample}_{\mathrm{o}}+\operatorname{coeff}_{14} * \operatorname{line}_{\mathrm{o}}+\operatorname{coeff}_{15} * \text { sample }_{\mathrm{o}} * \operatorname{line}_{\mathrm{o}} \\
\text { if producing AST_L1T then } \\
\text { sample }_{i}=\text { sample }_{i}+\text { terrain offset[sample][elev] }
\end{gathered}
$$

end if

Determine the integer and sub-pixel sample and line locations with total, equations 6466.

$$
\begin{gathered}
d s=\text { sample }_{i}-\text { isample } \\
\qquad d=\text { line }_{i}-\text { iline } \\
\text { Output pixel [line }][\text { sample }]=\text { total }
\end{gathered}
$$

end for

end for

\section{Resample ASTER L1A imagery}

Resample the ASTER Level 1 input image using the systematic grid and the terrain offset dataset, to produce terrain systematic images.

This resampling of the ASTER L1A image is only executed if the generation of the precision grid that occurs in the terrain precision correction process fails; for instance, when the scene is a night-time-only collection or the scene has a high amount of clouds.

\section{Terrain Precision Correction}

In this process, the systematic image resampling is conducted for SWIR band 4, if available. Otherwise, VNIR band 2 is resampled. This resampled band is correlated with Landsat Modified Moravec Interest Operator (MMIO) ground control points. This process begins with the down-sampling of the systematic image, when necessary, from 15 meter to the 30 meter reference GLS2000 GCPs using the GPYRAMID algorithm. The GCPCorrelate algorithm (Park and Schowengerdt, 1983) then correlates the ground control points, and generates line and sample offsets used to update the systematic grid to create the precision grid. Then, all bands of the ASTER Level 1A input imagery are resampled using the precision grid and the matching DEM to create the terrain precision image. 


\section{GPYRAMID}

The GPYRAMID algorithm is only used when the 30 meter SWIR reference band 4 is not available. This algorithm creates a 30 meter resolution equivalent of the 15 meter VNIR band 2 to the 30 meter GLS2000 GCP chips. In general, the GPYRAMID algorithm creates undersampled images using the Gaussian resampling technique at multiple resolutions (Adelson and others, 1984), also called pyramid levels. See figure 13 for an example image using the GPYRAMID algorithm.

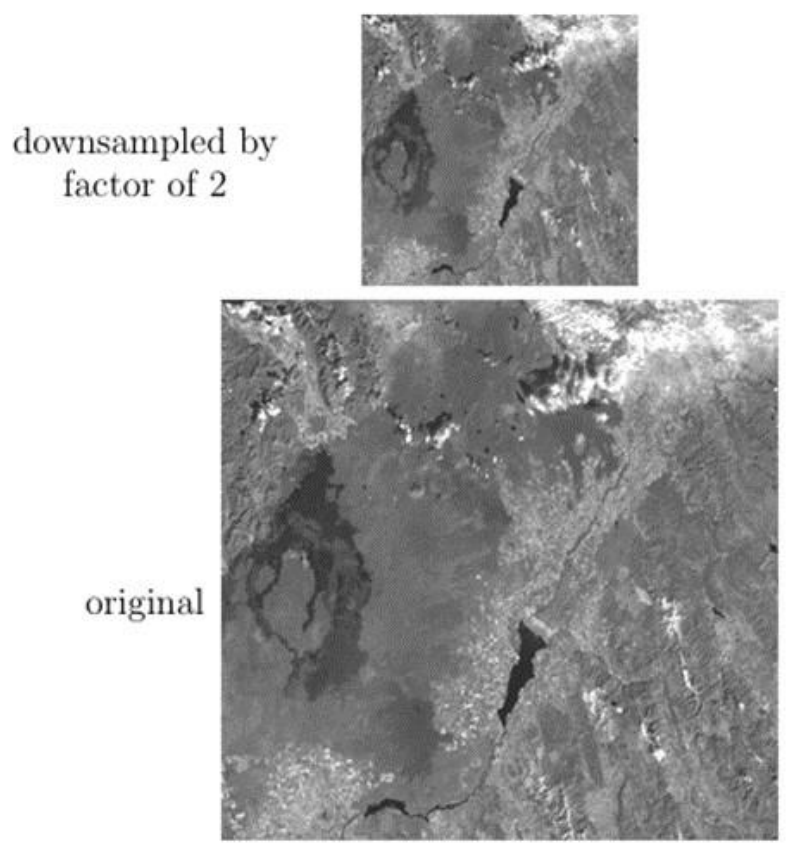

Systematic Image

Figure 13. Gaussian image pyramid level.

Correlate the Available Ground Control Points with the Systematic Image

The GCPs are small image chips (64x64 pixels) with geographic information that have been extracted from the reference image using the Modified Moravec Interest Operator (MMIO) algorithm developed to identify well-defined interest points from the reference scene (U.S. Geological Survey, Earth Resource Observation and Science Center, 2008). Using these interest points increases the success of correlation with the search image and provides accurate offsets. For AST_L1T processing, the Global Land Survey 2000 (GLS2000) dataset is used as reference image for the precision correction process. The USGS validated the GLS2000 reference dataset to have an expected root mean squared error (RMSE) of 25 meters or less (Rengarajan and others, 2015).

The GCPCorrelate is used to identify GCPs that are likely to be in error due to miscorrelation. It correlates the GLS2000 MMIO GCP chips (U.S. Geological Survey, Earth Resource Observation and Science Center, 2008) with the systematic image, computing the relative offsets measured at the pyramid level.

Unless otherwise specified, normalized cross-correlation measures the spatial differences between two image sources, one image source is considered the search image, whereas the other 
image source is considered the reference image. The correlation process only measures linear distortions over the windowed areas. By choosing windows (for example, chips) that are well distributed throughout the imagery, nonlinear differences between the image sources can be found.

Normalized cross-correlations produce a discrete correlation surface. To find a subpixel location associated with the offset, fit a polynomial around a 3x3 area centered on the correlation peak, and use the polynomial coefficients to solve for the peak or subpixel location. The normalized cross-correlation process reduces any correlation artifacts that may arise from radiometric differences between the two image sources.

If the two image windows of size $\mathrm{N} \times \mathrm{M}$ are defined by $f$ and $g$, the mensuration steps are as follows:

1. Perform normalized gray scale correlation using equation 67

$$
R(x, y)=\frac{\sum_{j=-\frac{N}{2}}^{\frac{N}{2}} \sum_{i=-\frac{M}{2}}^{\frac{M}{2}}[(f(j, i)-\bar{f})(g(x+j, y+i)-\bar{g})]}{\sum_{j=-\frac{N}{2}}^{\frac{N}{2}} \sum_{i=-\frac{M}{2}}^{\frac{M}{2}}\left[(f(j, i)-\bar{f})^{2}(g(x+j, i+i)-\bar{g})^{2}\right]^{\frac{1}{2}}}
$$

where equations $68-69$ solve for $\bar{f}$ and $\bar{g}$.

$$
\begin{gathered}
\bar{f}=\frac{1}{(M+1)(N+1)} \sum_{j=-\frac{N}{2}}^{\frac{N}{2}} \sum_{i=-\frac{M}{2}}^{\frac{M}{2}} f(j, i) \\
\bar{g}=\frac{1}{(M+1)(N+1)} \sum_{j=-\frac{N}{2}}^{\frac{N}{2}} \sum_{i=-\frac{M}{2}}^{\frac{M}{2}} g(x+j, y+i)
\end{gathered}
$$

2. Find the peak of the discrete correlation surface.

3. Fit a second order polynomial to a $3 \times 3$ area centered on the correlation peak. The polynomial takes the form of equation 70 .

$$
P(x, y)=a_{0}+a_{1} x+a_{2} y+a_{3} x y+a_{4} x^{2}+a_{5} y_{2}
$$

A least squares fit is performed on the points to solve for the polynomial coefficients. Set up matrices, equation 71 .

$$
[Y]=[X][a]
$$

where $X$ is a $9 \times 6$ matrix of correlation locations centered around the peak, $Y$ is $9 \times 1$ matrix of correlation values corresponding to $X$ locations, and $a$ is a 6 x 1 matix of polynomial coefficients.

D. Solve for polynomial coefficients using equation 72 .

$$
[a]=\left([X]^{T}[X]\right)^{-1}[X]^{T}[Y]
$$

E. Find partial derivatives of polynomial equation in terms of $\mathrm{x}$, equation 73 , and $\mathrm{y}$, equation 74 .

$$
\begin{aligned}
& \frac{\partial}{\partial x} P(x, y)=a_{1}+a_{3} y+2 a_{4} x \\
& \frac{\partial}{\partial y} P(x, y)=a_{1}+a_{3} x+2 a_{5} y
\end{aligned}
$$

4. Set partial equations equal to zero and solve for $\mathrm{x}$, equation 75 , and $\mathrm{y}$, equation 76 . 


$$
\begin{aligned}
& \mathrm{x}_{\text {offset }}=\frac{2 a_{1} a_{5}-a_{2} a_{3}}{a_{3}^{2}-4 a_{4} a_{5}} \\
& \mathrm{y}_{\text {offset }}=\frac{2 a_{2} a_{4}-a_{1} a_{3}}{a_{3}^{2}-4 a_{4} a_{5}}
\end{aligned}
$$

5. Combine the subpixel offset calculated in step 4 to the peak location from step 2 to calculate the total offset.

\section{Create Precision Grid}

The AST_L1T precision resampling grid consists of a set of evenly spaced output locations that are at integer sample and line locations. These integer and line locations map to a set of floating point, non-integer, input space sample and line coordinates. A set of four grid coordinates, two consecutive in the sample direction and two consecutive in the line direction, defines one grid cell. A group of bilinear polynomials, determined from the grid cell corner coordinates, map line and sample coordinates between input (ASTER Level 1) space to output (map projected) space.

The Refine algorithm generates the precision grid from the systematic grid using the registration information, such as GCP residuals. The Refine algorithm starts by using the GCPs $\mathrm{x}$ and y offset generated with the GCPCorrelate algorithm. Each of the GCPs is adjusted for relief displacement in the input image (ASTER L1A) using the systematic grid. The adjusted GCPs in the input image are projected back to the output grid space using the same systematic grid. The systematic image location of each GCP and its relief-adjusted correlated locations are used to fit the polynomial of either first or second order using the least squares fit method. The outliers are removed by comparing the residuals of the least squares fit to the weighted standard deviation. The systematic grid is adjusted with the polynomial coefficients to generate a precision grid, which relates the output projection location to the input line and sample location from the ASTER Level 1 image. The geometric resampling algorithm uses the precision grid to create a terrain precision corrected product. By default, the second order polynomial fit is used for precision correction. If significant warping occurs from the second order polynomial fit, then a first order polynomial fit is used for precision correction. To determine warping on the precision corrected image, check if the set of points along each edge of the precision-corrected image lies in a straight line to within a specified tolerance. The following section explains the Refine algorithm as implemented in S4PM.

1. Read the input data.

The necessary input to this algorithm, such as the systematic image's projection and geographic corner information, and the systematic grid file are read along with any other required ancillary information.

2. Find the line and sample locations for all GCPs in the input grid space, see figure 14. The systematic grid relates the input line and sample location from the ASTER Level 1 image to the output projection line and sample location. The grid structure stores the corresponding location of grid points in input space and output space, and the polynomial for each grid cell. The location of the GCPs in the input space is determined by finding the location of the GCPs in the appropriate grid cells and by using the reverse mapping coefficients.

3. Apply relief adjustment to GCPs. 
L1T space
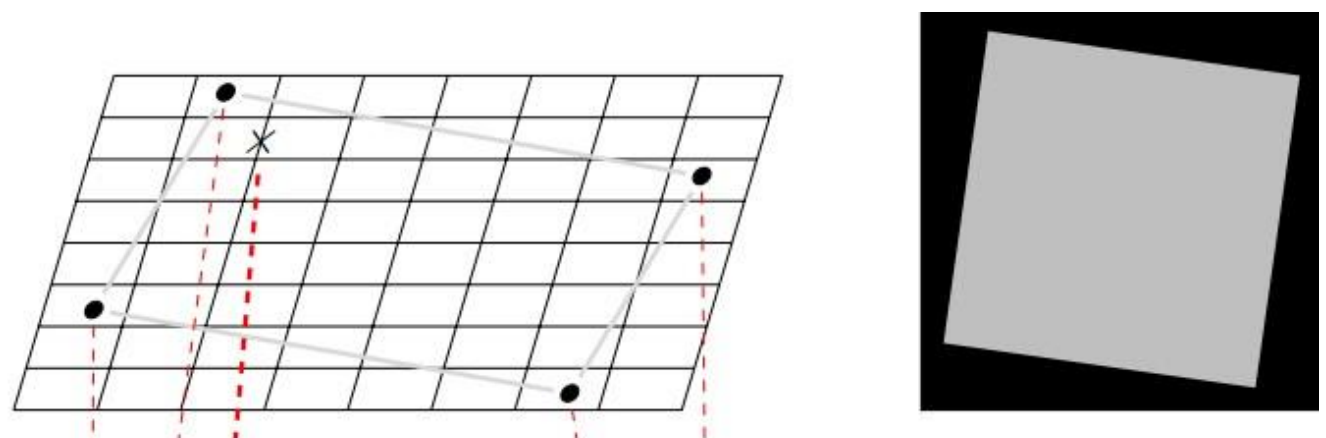

\section{L1B space}
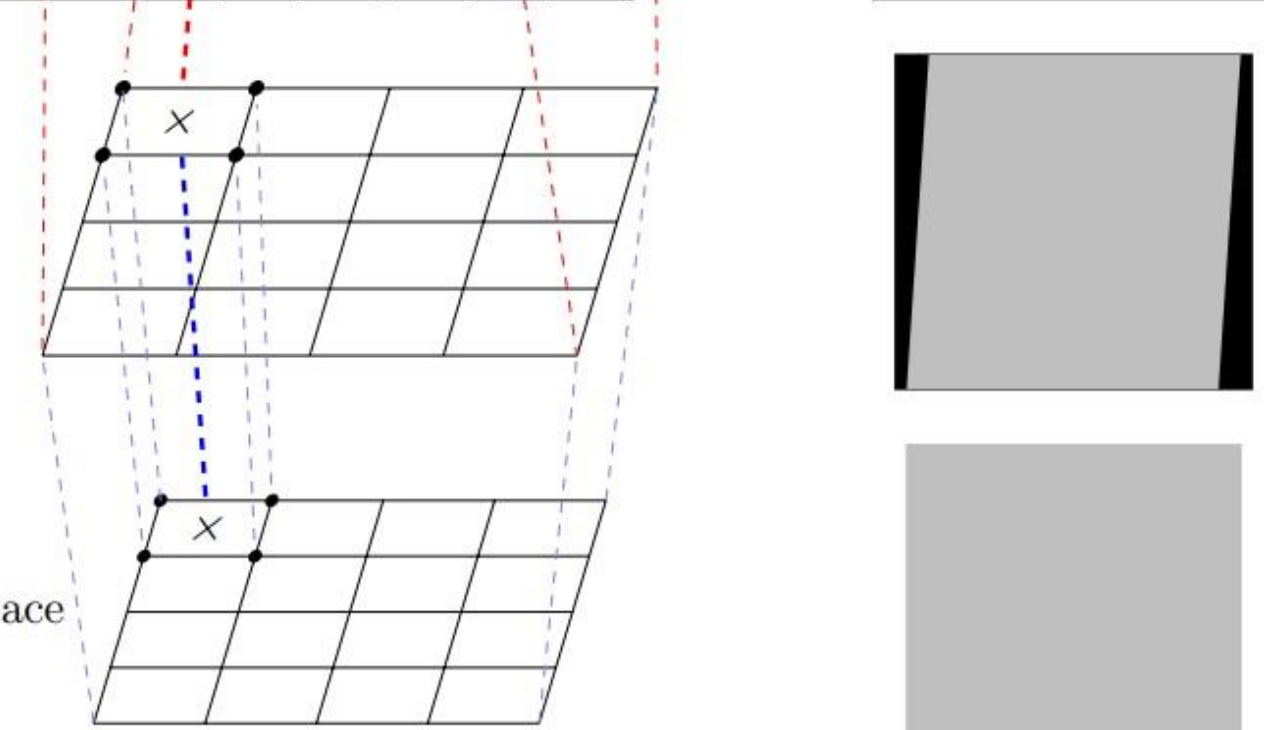

L1A space

Figure 14. Grid cell mapping.

4. The GCPs used for registration were derived from the GLS2000 imagery. To account for the parallax error because of terrain or relief, an adjustment in the LOS is required. To account for the relief adjustments, adjust the input sample location. The GCPs in the input space locations are adjusted based on the off-nadir across-track viewing angle and its corresponding elevation. The nadir sample from the previous step determines the offnadir across-track viewing angle. Use the following formulas in equations $77-85$ to determine the sample offset for adjustment due to relief

$$
\begin{gathered}
g_{r}=\text { PixelSize x }(\text { sample }- \text { nadirsample }) \\
\text { CentralAngle }=\alpha=\frac{g_{r}}{R_{e}}
\end{gathered}
$$

where $R_{e}$ is the radius of the Earth

$$
\operatorname{los}=\sqrt{R_{e}^{2}+\left(R_{e}+\text { alt }\right)^{2}-2 R_{e}\left(R_{e}+\text { alt }\right) \cos \mathrm{s}}
$$

where alt is the altitude of the spacecraft

$$
\beta=\sin -1\left(\frac{R_{e}}{l o s} \sin s\right)
$$




$$
d \theta=\tan ^{-1}\left(\frac{\operatorname{elev}\left(R_{e}+\text { alt }\right) \sin \beta / R_{e}}{\left(R_{e}+\text { elev }\right) \sqrt{1-\left(R_{e}+\text { alt }\right)^{2} \frac{\sin ^{2} \beta}{R_{e}^{2}}-\left(R_{e}+\text { alt }\right) \operatorname{cos~s}}}\right)
$$

where elev is the elevation of the ground control point

$$
z_{p p}=\sin ^{-1}\left(\left(R_{e}+\text { alt }\right) \sin \left((\beta+d \theta) / R_{e}\right)\right)
$$

where $z_{p p}$ is the exterior angle for the IFOV angles as a function of $\beta$ and $d \theta$ in radians

$$
d s=z_{p p}-s-\beta-d \theta
$$

where $d s$ is the sample center earth angle for current sample in radians

$$
d g_{r}=R_{e} d s
$$

where $d g_{r}$ is the arc length for the current sample and elevation in meters

$$
\text { sample }_{\text {adj }}=\text { sample }+\operatorname{dir} \frac{d g_{r}}{\text { PixelSize }}
$$

where dir equals 1 if the sample is less than the nadirsample, otherwise dir is equal to -1 .

5. Find the line and sample locations for all relief-adjusted GCPs in the output grid space. The same systematic grid structure finds the line and sample locations for all reliefadjusted GCPs in the output projection space.

6. Compute the polynomial fit coefficients.

The ASTER scenes are precision corrected using either the first order or second order polynomial function. The first order term includes the cross term, and is not truly a function with order 1 . The polynomial fit coefficients are computed to map between the systematic image location of each GCP and its relief-adjusted correlated location in the output projection space using the least squares fit method. The following methodology determines the polynomial fit coefficients.

The second degree fit polynomial equation is expressed as follows in equations 86-87.

$$
\begin{gathered}
\text { sample }_{a d j}=a_{0}+a_{1} \text { sample }+a_{2} \text { line }+a_{3} \text { line } * \text { sample }+a_{4} \text { sample }^{2}+a_{5} \text { line }^{2} \\
\text { line }_{\text {adj }}=b_{0}+b_{1} \text { sample }+b_{2} \text { line }+b_{3} \text { line } * \text { sample }+b_{4} \text { sample }^{2}+b_{5} \text { line }^{2}
\end{gathered}
$$

The first degree fit polynomial equation is expressed as follows in equations 88-89

$$
\begin{gathered}
\text { sample }_{\mathrm{adj}}=\mathrm{a}_{0}+\mathrm{a}_{1} \text { sample }+\mathrm{a}_{2} \text { line }+\mathrm{a}_{3} \text { line } * \text { sample } \\
\text { line }_{\mathrm{adj}}=\mathrm{b}_{0}+\mathrm{b}_{1} \text { sample }+\mathrm{b}_{2} \text { line }+\mathrm{b}_{3} \text { line } * \text { sample }
\end{gathered}
$$

where sample adj $_{\text {and line }}$ adj are a GCP's correlated and relief-adjusted line and sample location in the output projection space; sample and line are the GCPs location in the output projection space of the systematic grid, $a_{0}-a_{5}$ and $b_{0}-b_{5}$ are the second order polynomial fit coefficients, and $a_{0}-a_{3}$ and $b_{0}-b_{3}$ are the first order polynomial fit coefficients. 
These equations are solved using least squares adjustment in the matrix form using equation 90

$$
\text { [Predicted_line_sample][fit_coeff] }=\text { [true_line_sample }]
$$

or as in equations $91-92$

$$
\begin{aligned}
& {\left[A_{1}\right]\left[\operatorname{coeff}_{\mathrm{s}}\right]=\left[b_{s}\right]} \\
& {\left[A_{2}\right]\left[\operatorname{coeff}_{\mathrm{l}}\right]=\left[b_{l}\right]}
\end{aligned}
$$

where

$$
\begin{aligned}
& A_{1 n, 0}=1 \\
& A_{1 n, 1}=\text { sample }_{\mathrm{n}} \\
& A_{1 n, 2}=\text { line }_{\mathrm{n}} \\
& A_{1 n, 3}=\text { sample }_{\mathrm{n}} \text { line }_{\mathrm{n}} \\
& A_{1 n, 4}=\operatorname{sample}_{\mathrm{n}}^{2} \text { (if fit order }=2 \text { ) } \\
& A_{1 n, 5}=\text { line }_{\mathrm{n}}^{2} \text { (if fit order }=2 \text { ) } \\
& A_{2 n, 0}=1 \\
& A_{2 n, 1}=\text { sample }_{\mathrm{n}} \\
& A_{2 n, 2}=\text { line }_{\mathrm{n}} \\
& A_{2 n, 3}=\text { sample }_{\mathrm{n}} \text { line }_{\mathrm{n}} \\
& A_{2 n, 4}=\text { sample }_{\mathrm{n}}^{2} \text { (if fit order }=2 \text { ) } \\
& A_{2 n, 5}=\text { line }_{\mathrm{n}}^{2} \text { (if fit order }=2 \text { ) } \\
& b_{s n}=\left[\text { sample }_{\mathrm{adj}}\right]_{\mathrm{n}} \\
& b_{l n}=\left[\text { line }_{a d j}\right]_{n} \\
& \operatorname{coeff}_{\mathrm{s}}= \begin{cases}{\left[a_{0}, a_{1}, a_{2}, a_{3}, a_{4}, a_{5}\right]^{T}} & \text { (if fit order }=2 \text { ) } \\
{\left[a_{0}, a_{1}, a_{2}, a_{3}\right]^{T}} & \text { (if fit order }=1 \text { ) }\end{cases} \\
& \operatorname{coeff}_{1}= \begin{cases}{\left[b_{0}, b_{1}, b_{2}, b_{3}, b_{4}, b_{5}\right]^{T}} & \text { (if fit order }=2) \\
{\left[b_{0}, b_{1}, b_{2}, b_{3}\right]^{T}} & \text { (if fit order }=1)\end{cases}
\end{aligned}
$$

$n$ is the index of the GCPs, $0 \leq n<k$ where $k$ is the number of GCPs.

7. Outlier detection and removal.

The polynomial fit is iterated several times until the number of iterations reaches the maximum number allowed or no outliers are found. A GCP is detected as an outlier if its residuals, after applying the polynomial fit, are greater than the fit threshold. To determine the fit thresholds, sum the squares of the residuals obtained during polynomial fit, equations 93-96, in the line and sample direction. These sum squared residuals are weighted by a tolerance to calculate the fit threshold, equation 97. Points whose residuals, after polynomial fit are greater than the fit thresholds, are marked as outliers and removed; the remaining points are reiterated for new polynomial fit coefficients as in equation 98 .

$$
\begin{gathered}
{\left[\text { residual }_{\text {sample }}\right]=\left[A_{1}\right]\left[\operatorname{coeff}_{\mathrm{s}}\right]-\left[b_{1}\right]} \\
{\left[\text { residual }_{\text {line }}\right]=\left[A_{2}\right]\left[\operatorname{coeff}_{\mathrm{l}}\right]-\left[b_{2}\right]}
\end{gathered}
$$




$$
\begin{aligned}
& \operatorname{res}_{\mathrm{s}}^{2}=\sum \text { residual }_{\text {sample }}^{2} \\
& \operatorname{res}_{1}^{2}=\sum \text { residual }_{\text {line }}^{2} \\
& \text { threshold }{ }_{\text {fit }}=\text { tolerance } \sqrt{\frac{\operatorname{res}_{\mathrm{s}}^{2}+\mathrm{res}_{1}^{2}}{\mathrm{num}_{\mathrm{gcp}}}} \\
& \text { residuals }_{\mathrm{i}}=\sqrt{\text { residual }_{\text {sample }_{\mathrm{i}}}^{2}+\text { residual }_{\text {line }}^{2}}
\end{aligned}
$$

If residuals $\mathrm{i}_{\mathrm{i}}$ is greater than thresholdfit, then $\mathrm{GCP}_{\mathrm{i}}$ is an outlier where $i$ is the index of the GCP, residual sample $_{\mathrm{i}}$ is the $i$ th index of the [residual $_{\text {sample }] \text { array, residual }}$ line $_{\mathrm{i}}$ is the $i$ th index of the [residual ${ }_{\text {line }}$ ] array, tolerance is a defined number, and num ${ }_{\mathrm{gcp}}$ is the number of GCPs.

8. Generate the precision grid.

A. Adjust the grid framing.

As an initial approximation, the systematic grid is copied as a precision grid. The four corner coordinates of the grids are adjusted with the zero order fit polynomial coefficients. This step ensures that the precision grid frame covers the whole image, and is not clipped due to the systematic scene's offset from its true geographic location. Because the grid framing coordinates were adjusted, the line and sample values also need adjustment. To adjust these values, add the line(l) and sample(s) offsets to each of the line and sample location coordinates in the output space of the grid. In general, these values are indexed such that the first pixel has the coordinate $(0,0)$ in the output space. At this stage, the precision grid is adjusted to account for the frame shift, which prevents any image from being clipped in the final product.

B. Scale output space for different pixel sizes is implemented using equations 99-106,

$$
\begin{aligned}
& \text { if (ls_to_line[0] > 0.0) } \\
& \mathrm{l}_{\text {offset }}=(\text { int })(- \text { ls_to_line }[0] * \text { largest_pixsize_scale }-0.5) \text {; } \\
& \text { else } \\
& \mathrm{l}_{\text {offset }}=(\text { int })(- \text { ls_to_line }[0] * \text { largest_pixsize_scale }+0.5) \text {; } \\
& \text { if (ls_to_samp[0] > 0.0) } \\
& \mathrm{s}_{\text {offset }}=(\text { int })(- \text { ls_to_samp[0] } * \text { largest_pixsize_scale }-0.5) \text {; } \\
& \text { else } \\
& \mathrm{s}_{\text {offset }}=(\mathrm{int})(- \text { ls_to_samp[0] } * \text { largest_pixsize_scale }+0.5) \text {; } \\
& \mathrm{l}_{\text {offset }} *=\text { inv_current_largest_pixsize_scale; } \\
& \mathrm{s}_{\text {offset }} *=\text { inv_current_largest_pixsize_scale; }
\end{aligned}
$$

where 1 is line and s means sample.

Keep x/y offset the same for all bands using equations 107-116

if (source_band_flag) 


$$
\begin{gathered}
\mathrm{x}_{\mathrm{offset}}=\mathrm{s}_{\mathrm{offset}} * \text { pixsize; } \\
\mathrm{y}_{\text {offset }}=-\mathrm{l}_{\text {offset }} * \text { pixsize; }
\end{gathered}
$$

\}

$$
\begin{gathered}
\text { prec_grid. corners. upleft. } \mathrm{x}+=\mathrm{x}_{\mathrm{offset}} \\
\text { prec_grid. corners. upleft. } \mathrm{y}+=\mathrm{y}_{\mathrm{offset}} \\
\text { prec_grid. corners. upright. } \mathrm{x}+=\mathrm{x}_{\mathrm{offset}} \\
\text { prec_grid. corners. upright. } \mathrm{y}+=\mathrm{y}_{\mathrm{offset}} \\
\text { prec_grid. corners. loleft. } \mathrm{x}+=\mathrm{x}_{\mathrm{offset}} \\
\text { prec_grid. corners. loleft. } \mathrm{y}+=\mathrm{y}_{\mathrm{offset}} \\
\text { prec_grid. corners. loright. } \mathrm{x}+=\mathrm{x}_{\mathrm{offset}} \\
\text { prec_grid. corners. loright. } \mathrm{y}+=\mathrm{y}_{\mathrm{offset}}
\end{gathered}
$$

Find the precision input space line, sample coordinates for the grid using equations 117122.

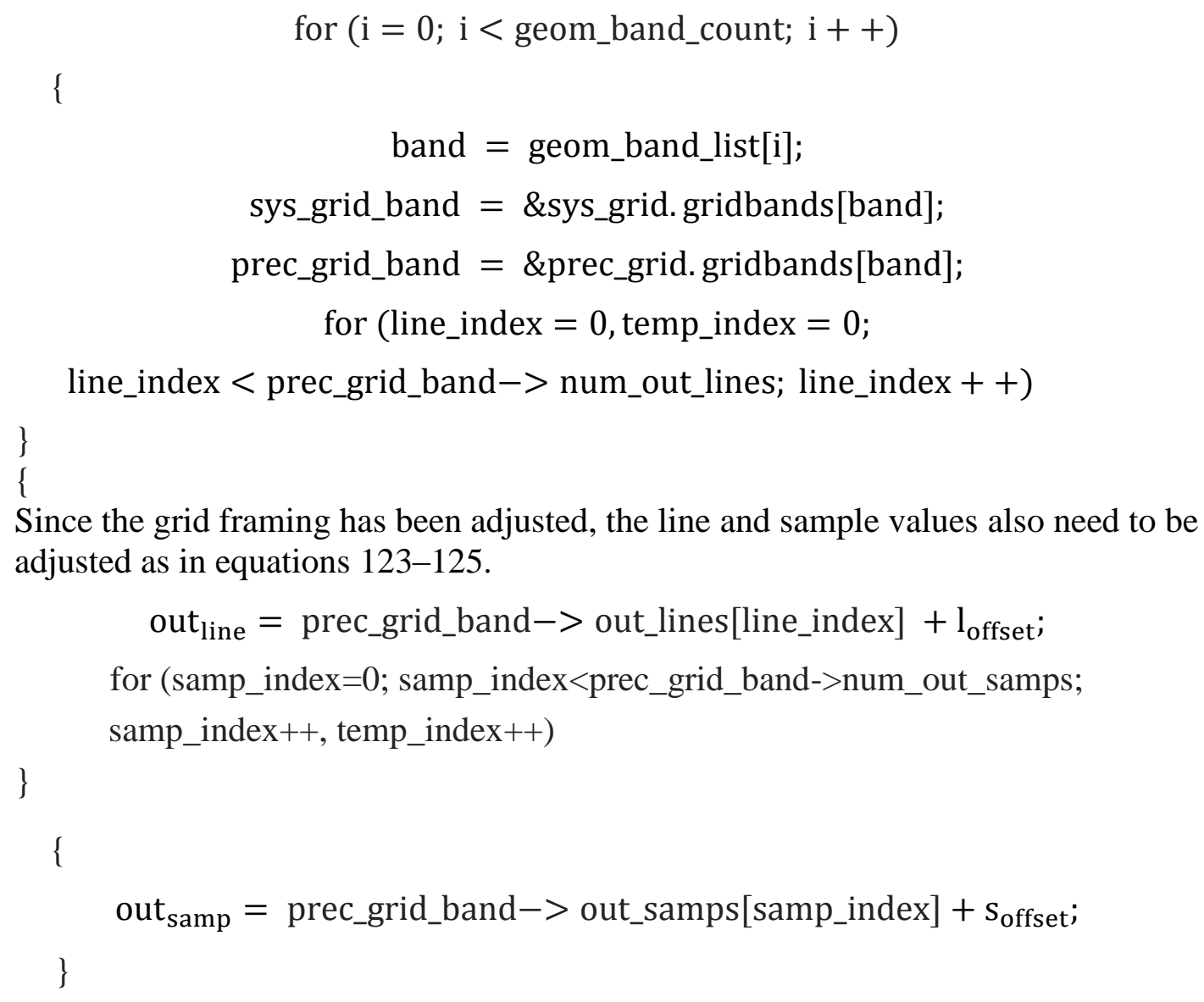$$
\text { for }(\mathrm{i}=0 \text {; } \mathrm{i}<\text { geom_band_count; } \mathrm{i}++ \text { ) }
$$$$
\text { band }=\text { geom_band_list }[\mathrm{i}] \text {; }
$$$$
\text { sys_grid_band = \&sys_grid.gridbands[band]; }
$$$$
\text { prec_grid_band = \&prec_grid.gridbands[band]; }
$$$$
\text { for (line_index }=0 \text {, temp_index }=0 \text {; }
$$$$
\text { line_index }<\text { prec_grid_band- }>\text { num_out_lines; line_index }++ \text { ) }
$$$$
\}
$$$$
\text { \{ }
$$

Since the grid framing has been adjusted, the line and sample values also need to be adjusted as in equations 123-125.$$
\text { out } \left._{\text {line }}=\text { prec_grid_band-> out_lines[line_index }\right]+\mathrm{l}_{\text {offset }} \text {; }
$$$$
\text { for (samp_index }=0 \text {; samp_index }<\text { prec_grid_band->num_out_samps; }
$$$$
\text { samp_index++, temp_index++) }
$$$$
\text { \} }
$$$$
\text { out } \left._{\text {samp }}=\text { prec_grid_band-> out_samps[samp_index }\right]+\mathrm{s}_{\text {offset }} \text {; }
$$
\} 


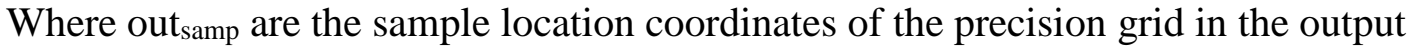

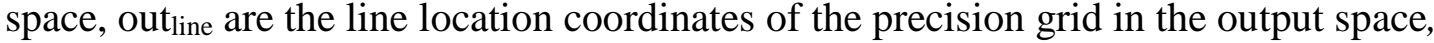
out $_{\text {samps }}$ are the sample location coordinates of the systematic grid in the output space, and out_lines are the line location coordinates of the systematic grid in the output space.

C. Calculate the precision grid coefficients for each grid cell.

The precision grid from the previous step is not accounting for the actual precision coefficients but only adjusted for framing. To adjust each output line and sample in the output space of the grid, use the polynomial fit coefficient equations to determine their output space coordinates. Once the grid point's output space coordinate is determined, use the systematic grid's input-output relationship (grid coefficients) to determine the corresponding input line and sample for the given grid point, see equations 126-137,

$$
\begin{aligned}
& \text { new_line }=\text { pixsize_scale } * \text { ls_to_line[0] } \\
& \text { + ls_to_line } * \text { out }_{\text {line }} \\
& \text { + ls_to_line } * \text { out }_{\text {samp }} \\
& \text { + inv_pixsiz_scale } * \text { ls_to_line[3] } * \text { out }_{\text {line }} * \text { out }_{\text {samp }} \\
& \text { + inv_pixsiz_scale } * \text { ls_to_line[4] } * \text { out }_{\text {line }} * \text { out_line }
\end{aligned}
$$

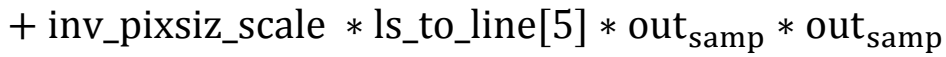

$$
\begin{aligned}
& \text { new_samp }=\text { pixsize_scale } * \text { ls_to_samp[0] } \\
& \text { + ls_to_samp[1] } * \text { out }_{\text {line }} \\
& \text { + ls_to_samp[2] } * \text { out }_{\text {samp }} \\
& + \text { inv_pisize_scale } * \text { ls_to_samp[3] } * \text { out }_{\text {line }} * \text { out }_{\text {samp }} \\
& \text { + inv_pixsize_scale } * \text { ls_to_samp[4] } * \text { out }_{\text {line }} * \text { out }_{\text {line }} \\
& \text { + inv_pixsize_scale } * \text { ls_to_samp[5] } * \text { out }_{\text {samp }} * \text { out }_{\text {samp }}
\end{aligned}
$$

where new_samp are the new sample coordinates of the precision grid in the output grid space and new_line are the new line coordinates of the precision grid in the output grid space. The systematic grid cell coefficients determine the input line and sample location for the corresponding precision line and sample grid coordinates (new_samp and new_line). For each grid cell point in the output space, their corresponding input line and sample locations are determined. Each grid cell's corner and central points determine bilinear fit coefficients.

D. Check for scene warping.

The precision correction process uses second order polynomial functions, which could cause skew or warp the image if the points used for fitting the polynomials are not accurate or if the systematic models are not accurate. Because of the nature of the second order polynomial fit, testing is required to ensure that there is no skew or warping observed in the precision-corrected image. If skew is detected in the scene, then the Refine algorithm uses first order polynomial fit for precision correction. If a scene is 
precision corrected using the first order polynomial fit, then the scene curvature tests are skipped, and the results from the first order fit generate the precision grid.

E. Find the input and output grid points.

To validate the curvature of the scene edges, select a few grid points on the top, bottom, left, and right edges of the image (from grid). The distance of these grid points to the straight line connecting the first and last grid point for each of the four directions (top, left, right, and bottom) are determined ( $d$ as shown in figure 15). If the distance measured $(d)$ exceeds the tolerance, then the image is assumed to have warping, and falls back to first order polynomial fit correction.

The grid points are selected in the input space of the precision grid such that the grid points are equidistant from each other along the edges of the image. In figure 15, seven grid points are used for the curvature test of which, two points are the first and last points along the edge. To determine each of these points' corresponding location in the output space of the precision grid, use the precision grid coefficients and the corresponding grid cells. To construct a straight-line equation, use the first and last point's location in the output grid space for each edge (top, left, bottom, and right). The distance between each curvature point to its corresponding straight line is then calculated. If the distance $\left(d_{i}\right)$ values are beyond the curvature tolerance $\left(t_{i}\right)$ then the precision-corrected scene fails the curvature test and the precision correction process continues with the first order polynomial fit correction.

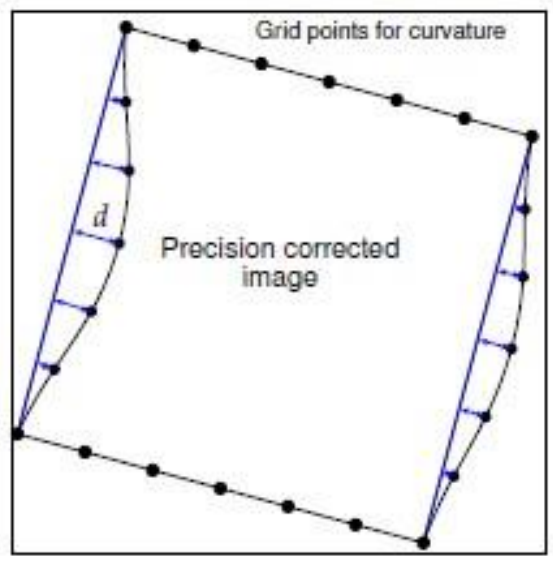

Figure 15. Curvature test for precision correction process.

for all curvature test points do

if $d_{i}>t_{i}$ then

Precision corrected scene likely to have warping.

Scene corrected with first order polynomial fit. end if

end for

The generated precision grid produces a precision corrected product.

\section{Resample DEM Using the Precision Grid}

Here, the DEM is resampled to match the precision grid. 
for line $=0$ to number of output lines do

for sample $=0$ to number of output samples do

if producing AST_L1T then

Extract the corresponding elevation from the DEM.

Map the AST_L1T line/sample to the corresponding (path oriented) ASTER

Level 1 line/sample using the grid.

Use the elevation and the ASTER Level 1 line sample to look up the sample offset from the terrain table.

Interpolate the output pixel value at the calculated Level 1A coordinates.

end if

Determine grid cell number for output pixel location.

Read the grid cell reverse mapping coefficients for the grid cell number.

Calculate the input line and sample location as in equations 138-139.

sample $_{\mathrm{i}}=\operatorname{coeff}_{8}+\operatorname{coeff}_{9} *$ sample $_{\mathrm{o}}+\operatorname{coeff}_{10} * \operatorname{line}_{\mathrm{o}}+\operatorname{coeff}_{11} *$ sample $_{\mathrm{o}} * \operatorname{line}_{\mathrm{i}}$

line $_{\mathrm{i}}=\operatorname{coeff}_{12}+\operatorname{coeff}_{13} *$ sample $_{\mathrm{o}}+\operatorname{coeff}_{14} * \operatorname{line}_{\mathrm{o}}+\operatorname{coeff}_{15} *$ sample $_{\mathrm{o}} *$ line $_{\mathrm{i}}$

if producing AST_L1T then use equation 140

$$
\text { sample }_{i}=\text { sample }_{i}+\text { terrain offset }[\text { sample] }[\mathrm{elev}]
$$

end if

Determine the integer and subpixel line and sample locations as in equations 141-142

$$
\begin{gathered}
d s=\text { sample }_{\mathrm{i}}-\text { isample } \\
d l=\text { line }_{\mathrm{i}}-\text { iline }
\end{gathered}
$$

where isample $=\left[\right.$ sample $\left._{\mathrm{i}}\right]$ and iline $=\left[\right.$ line $\left._{\mathrm{i}}\right]$.

\section{Resample ASTER L1A image}

A single resampling of the ASTER Level 1A image using the terrain dataset and the newly generated precision grid, produces the terrain precision images that are corrected to their absolute position.

\section{Geometric Verification}

The geometric verification algorithm, referred to as GVERIFY, determines the relative accuracy of the terrain and precision corrected scene when compared to the corresponding orthorectified GLS2000 scene.

The geometric verification algorithm uses a cross-correlation procedure along with a simple outlier detection algorithm to determine the relative offsets of the terrain corrected scene to the reference GLS2000, which are accurate to 25 meters positional accuracy (Rengarajan and others, 2015). The expectation of this algorithm is to provide a relative error estimate for four quadrants of the scene, overall relative error estimate of the full scene, and to provide a colorcoded browse image showing the relative offsets at different geographic locations within the scene. Because of the inherent nature of the cross-correlation process and scene content issues 
within the ASTER scene, limitations exist for this algorithm, which are described at the end of this section.

The inputs to this algorithm are an AST_L1T corrected scene in Landsat HDF format and the corresponding reference orthorectified GLS2000 products. A dense set of evenly spaced GCP locations and corresponding data are extracted from the search image (precision terrain corrected product); these geographic locations are transferred to the reference GLS2000 image, where a second set of reference GCPs are extracted. The reference image is correlated with the search image and the correlation statistics (peak and strength) are compared against their corresponding thresholds, which were derived by empirical methods. All correlated points that pass the minimum thresholds are used for further calculation and outlier rejection. For all points, radial offsets are calculated by the combined RMSE of the offsets in line and sample direction.

The outlier detection algorithm uses a neighborhood analysis only for points whose radial offsets are greater than 2 pixels (suspect points). For each suspect point, a bounding rectangular region is selected. The ratio for the number of points within the bounding box whose radial offsets are less than 2 pixel offsets to the total number of points is found and compared against a threshold derived from empirical analysis. If the ratio is greater than the threshold, then the suspect point is marked as an outlier. Irrespective of whether the point is an outlier or not, the ratios are used for other suspect point neighborhood analysis, and once all the outliers are detected, they are removed from the point set.

The last outlier detection algorithm uses a simple standard deviation based test. Points whose radial offsets are more than ' $n$ ' number of standard deviations from the mean, are considered as outliers. The final set of points after the standard deviation test is considered the set of good GCPs. Each point is ranked depending on its offsets.

For example:

Rank 1 Offsets less than 0.5 pixel

Rank 2 Offsets between 0.5 to 1 pixel

Rank 3 Offsets between 1 to 2 pixels

Rank 4 Offsets between 2 to 3 pixels

Rank 5 Offsets greater than 3 pixels

Simple statistics, such as mean, median, standard deviation, and RMSE for the good GCPs, are calculated for the entire scene, by dividing the scene into four regions. The set of good GCP points are color-coded based on their ranks, overlaid on the corrected product, and a browse image is generated. The quadrant RMSE and full scene RMSE are provided in the AST_L1T quality assurance (QA) files for the end user. The geometric algorithm uses all the required parameters from the Object Definition Language (ODL) files contained within the product generation executable (PGE). The algorithm and the implementation flow are provided in the following:

1. If the input scene UTM zone does not match that of the GLS reference image, reproject the input band into the reference image zone.

2. If the input band is 15 meter resolution (VNIR band 2), resample it to 30 meters to match the GLS2000 data resolution.

3. Generate an evenly spaced grid of GCP chip locations from the search image (ASTER L1A input scene). 
4. Using the geolocation information from the reference and search image and the search image GCP locations, the corresponding GCP locations in the reference image are determined. A GCP text file containing the geographic locations of the GCPs and their attributes such as pixel size, line, and sample locations, and so on, is then created using equations 143-146

$$
\begin{aligned}
& \text { line }_{\text {offset }}=\text { round }\left(\frac{-\left(\operatorname{search}_{\mathrm{UL}} \mathrm{y}-\text { ref }_{\mathrm{UL}}\right)}{\text { ref_proj_dist }_{\mathrm{y}}}\right) \\
& \text { sample }_{\text {offset }}=\text { round }\left(\frac{-\left(\operatorname{search}_{U_{\mathrm{X}}}-\mathrm{ref}_{\mathrm{UL}_{\mathrm{X}}}\right)}{\text { ref_proj_dist }_{\mathrm{X}}}\right)
\end{aligned}
$$

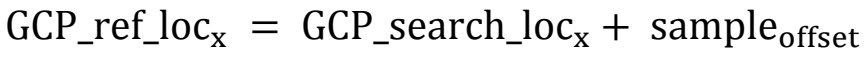

$$
\begin{aligned}
& \text { GCP_ref_loc } y=\text { GCP_search_loc } y+\text { line }_{\text {offset }}
\end{aligned}
$$

where

search_UL $L_{y}$ is the Upper Left line coordinate of the search image search_ULx is the Upper Left sample coordinate of the search image ref_ULy is the Upper Left line coordinate of the reference image ref_ULx is the Upper Left sample coordinate of the reference image ref_proj_dist $\mathrm{x}_{\mathrm{x}}$ is the projection distance for the reference image in sample direction ref_proj_dist $\mathrm{y}_{\mathrm{y}}$ is the projection distance for the reference image in line direction GCP_search_loc $c_{\mathrm{x}}$ is the GCP chip sample location in the search image GCP_search_loc $\mathrm{y}_{\mathrm{y}}$ is the GCP chip line location in the search image GCP_ref_loc ${ }_{\mathrm{x}}$ is the GCP chip sample location in the reference image, and GCP_ref_loc $y$ is the GCP chip line location in the reference image.

5. Perform cross-correlation using the GCP text file and store the residuals in a file (U.S. Geological Survey, 2006).

6. For each correlated point in step 5, determine whether the correlation peak value is greater than the correlation peak and if the correlation strength is greater than the correlation strength threshold. Note: The ODL file contains both of these thresholds.

7. Points that failed step 6 are marked as outliers and removed.

8. Use the formula in equation 147 to calculate a combined offset (radial) for each good point

$$
\mathrm{RMSE}_{\mathrm{r}}=\sqrt{\mathrm{res}_{\mathrm{l}}^{2}+\mathrm{res}_{\mathrm{s}}^{2}}
$$

where $\mathrm{res}_{\mathrm{l}}$ is the measured residuals in line direction, $\mathrm{res}_{\mathrm{s}}$ is the measured residuals in the sample direction, and $\mathrm{RMSE}_{\mathrm{r}}$ is the combined offset in line and sample direction.

9. Identify points as suspects where $\mathrm{RMSE}_{\mathrm{r}}$ is greater than the threshold for suspects as listed in the ODL.

10. If the number of suspect points identified in step 9 exceeds 50 percent of all points in step 8, then calculate the mean, median, standard deviation, RMSE, and Median Absolute Deviation (MAD) statistics for all points and continue with the neighbor analysis in step 12. If the number of suspect points are less than 50 percent of all points in step 7 , then 
calculate the MAD, the Z-value Median Absolute Deviation (or ZMAD), and median for all the points from step 7 using equations 148-149.

$$
\begin{gathered}
\text { MAD }=\text { med } \mid x_{i}-\text { med } x \mid \\
Z_{M A D}=\frac{\mid x_{i}-\text { med } x \mid}{\text { MAD }}
\end{gathered}
$$

11. Identify outlier points using ZMAD statistics and remove the outliers. Calculate the mean, median, standard deviation, RMSE, and MAD statistics for the good points (excluding outliers) ZMADi $>n$ where $n$ is a ZMAD outlier threshold from the ODL file.

12. Neighborhood analysis-For each suspect point (any points whose $\mathrm{RMSE}_{r}$ is greater than or equal to 2 pixels), construct a bounding box using equations 150-151, with the suspect point at the center,

$$
\begin{gathered}
\mathrm{UL}_{\text {box }}=\left[\mathrm{GCP}_{\mathrm{s}}-\text { dist }_{\mathrm{x}}, \mathrm{GCP} 1-\text { dist }_{\mathrm{y}}\right] \\
\mathrm{LR}_{\text {box }}=\left[\mathrm{GCP}_{\mathrm{s}}+\operatorname{dist}_{\mathrm{x}}, \mathrm{GCP} 1+\text { dist }_{\mathrm{y}}\right]
\end{gathered}
$$

where $\mathrm{GCP}_{s}$ is the sample location of the $\mathrm{GCP}$ in the search image, $\mathrm{GCP}_{l}$ is the line location of the GCP in the search image, dist $x_{x}$ is the distance in sample direction for

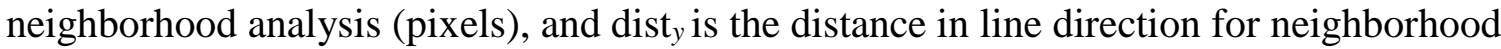
analysis (pixels). A sample distribution in the neighborhood of a suspect point is displayed in figure 16.

A. In the first iteration, the bounding box region is constructed with 200 pixels on either side of the suspect point.

B. Identify the number of points in this neighborhood (bounding box) that are considered valid from step 11.

C. If the number of points found in step $12 \mathrm{~B}$ is greater than or equal to 10 , then proceed to step 13.

D. If the number of points is less than 10, then increase the region size by a step size of 50 pixels. Repeat the step increase until either 10 points are found in the neighbor region or a maximum window size, provided in the ODL, is reached, whichever happens first.

E. If the number of points from step $12 \mathrm{D}$ is less than 5 , the suspect point under consideration is marked as an outlier but still considered for other suspect points' neighborhood analysis.

13. Find the number of correlated points in the bounding box; the number of points whose $\mathrm{RMSE}_{\mathrm{r}}<2$ pixels and the ratio between the two. To determine the number of points inside the bounding box, check each point's line and sample location to see if they are inside the bounding box line and sample locations. For each of those points inside the bounding box, the points whose RMSEr is less than 2 pixels are considered good points. The sample distribution displayed in figure 16 shows seven good points, three suspect points, and has a ratio of 0.7 .

14. If the ratio of good points to total points inside the bounding box is greater than the neighbor threshold (from ODL file), then the suspect point is flagged as an outlier. Otherwise, the suspect point is not considered an outlier. When suspect points are flagged as outliers, they are still considered suspect points for a different suspect points' 
neighborhood analysis. After running the neighborhood analysis for all suspect points in the scene, all points flagged as outliers are removed.

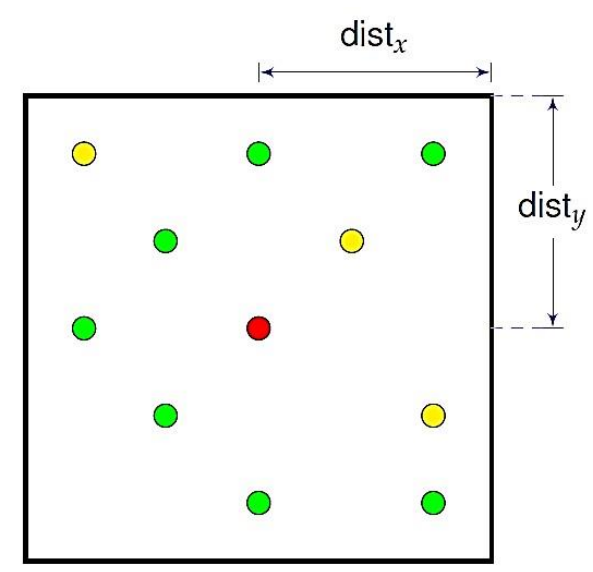

Suspect point under consideration for outlier

Suspect point (RMSE_LS $>2$ pixels)

Suspect point (RMSE_LS $<2$ pixels)

Figure 16. Neighborhood analysis.

15. Calculate the standard deviation, mean, median, RMSE, and MAD statistics for all non-outlier (valid) points from the previous step using equations 152-155:

$$
\begin{aligned}
& \mu=\frac{1}{n} \sum_{k=1}^{n} \mathrm{RMSE}_{r k} \\
& \sigma=\frac{1}{n-1} \sum_{k=1}^{n}\left(\mathrm{RMSE}_{\mathrm{rk}}-\mu\right)^{2} \\
& \text { med RMSE } r=\left\{\begin{array}{c}
\frac{1}{2}\left(\operatorname{RMSE}_{\mathrm{rs}\left(\frac{\mathrm{n}}{2}\right)}+\operatorname{RMSE}_{\mathrm{rs}\left(\frac{\mathrm{n}}{2}+1\right)}\right) \\
\operatorname{RMSE}_{\mathrm{rs}\left(\frac{\mathrm{n}+1}{2}\right)}
\end{array} \text { if } n\right. \text { is even } \\
& \mathrm{MAD}=\operatorname{med}\left|\mathrm{RMSE}_{\mathrm{rk}}-\mathrm{RMSE}_{\mathrm{r}}\right|
\end{aligned}
$$

where $n$ is the number of points, $\mu$ is the mean value of $\mathrm{RMSE}_{\mathrm{r}}$ for all $n$ points, $\sigma$ is the standard deviation of $\mathrm{RMSE}_{\mathrm{r}}$ for all $n$ points, med $\mathrm{RMSE}_{\mathrm{r}}$ is the median value for the $\mathrm{RMSE}_{\mathrm{r}}, \mathrm{RMSE}_{\mathrm{rk}}$ is the $k$ th index of $\mathrm{RMSE}_{\mathrm{r}}, \mathrm{RMSE}_{\mathrm{rs}}$ is $\mathrm{RMSE}_{\mathrm{r}}$ sorted by residuals, and $\mathrm{RMSE}_{\mathrm{rs}(n / 2)}$ is the $n / 2$ index of RMSE $\mathrm{R}_{\mathrm{rs}}$.

16. For each point, check if the ZMAD of that point is greater than the ZMAD threshold after the neighborhood analysis (comes from the ODL). If the ZMAD is greater, the identified points are considered outliers.

17. Generate the final statistics such as mean, median, RMSE, standard deviation, and MAD for all valid points after step 16. This is the full scene statistics.

18. The valid GCPs are ranked based on $\mathrm{RMSE}_{\mathrm{r}}$ depending on the ranks defined in the ODL. For example (in pixels):

$$
\begin{array}{ll}
\mathrm{RMSE}_{r} \leq 0.5 & \text { Rank 1 } \\
0.5<\mathrm{RMSE}_{r} \leq 1 & \text { Rank 2 }
\end{array}
$$




$$
\begin{array}{ll}
1<\mathrm{RMSE}_{r} \leq 2 & \text { Rank 3 } \\
2<\mathrm{RMSE}_{r} \leq 3 & \text { Rank 4 } \\
\operatorname{RMSE}_{r}>3 & \text { Rank 5 }
\end{array}
$$

19. The scene is divided into rectangular regions. The numbers of regions are defined in the ODL parameter for the geometric verification algorithm. For example, the scene in figure 17 is divided by three regions along the line and three regions along the sample direction. For standard processing the four quadrant regions $(2 \times 2)$ are established.
A. Determine the four vertices of the image location in the product frame.
B. Construct the equations for the lines connecting all four corners using the vertices.
C. Determine the spacing distance in line and sample direction by dividing the line and sample distance by the number of divisions along the line and sample direction.
D. Determine the vertices for each region using the starting coordinate (UL,LL), ending coordinate (UR,LR), slope, and intercept of the line from the left edge, right edge, top, and bottom edge of the image along with sample and line distances.

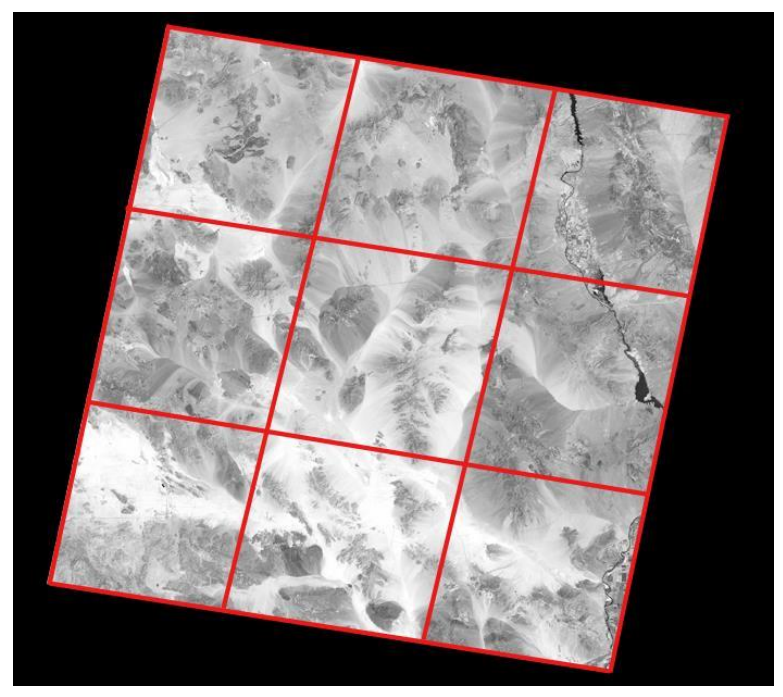

Figure 17. Scene divided into $3 \times 3$ regions.

20. For each region, find all points that fall within the region and their statistics such as mean, standard deviation, RMSE, median for line and sample, and radial directions, number of points, and histogram of ranks.
A. To determine the points inside the region, check whether the line and sample coordinate falls between the region's corner line and sample coordinates.
B. The point statistics such as mean, standard deviation, and RMSE are all calculated by formulas as shown in step 15.

21. To determine the quadrant statistics and full scene statistics, follow the same procedure as listed in steps 19 and 20.

22. For the quadrant statistics, the regions are generated such that there are two regions in the line and two regions in the sample direction. This method generates a quadrant and the statistics within the quadrant are determined as in step 20. 
23. Each valid GCP is assigned a color based on the rank, as in step 18 using a color LUT. These points are overlaid on the single band of the terrain precision or terrain systematic corrected image to show the distribution of valid GCPs and their color-coded radial offsets across the image.

24. To create a browse jpeg image of the valid GCP overlaid on an AST_L1T single band image, a three band Hierarchical Data Format (HDF) dataset (input from step 22) is generated with the GCPs overlain. This HDF dataset is converted into a portable network graphics (PNG) file, which is then reduced in size and converted to a jpeg image. An example of a well-registered ASTER L1T geometrically verified image is displayed in figure 18 .

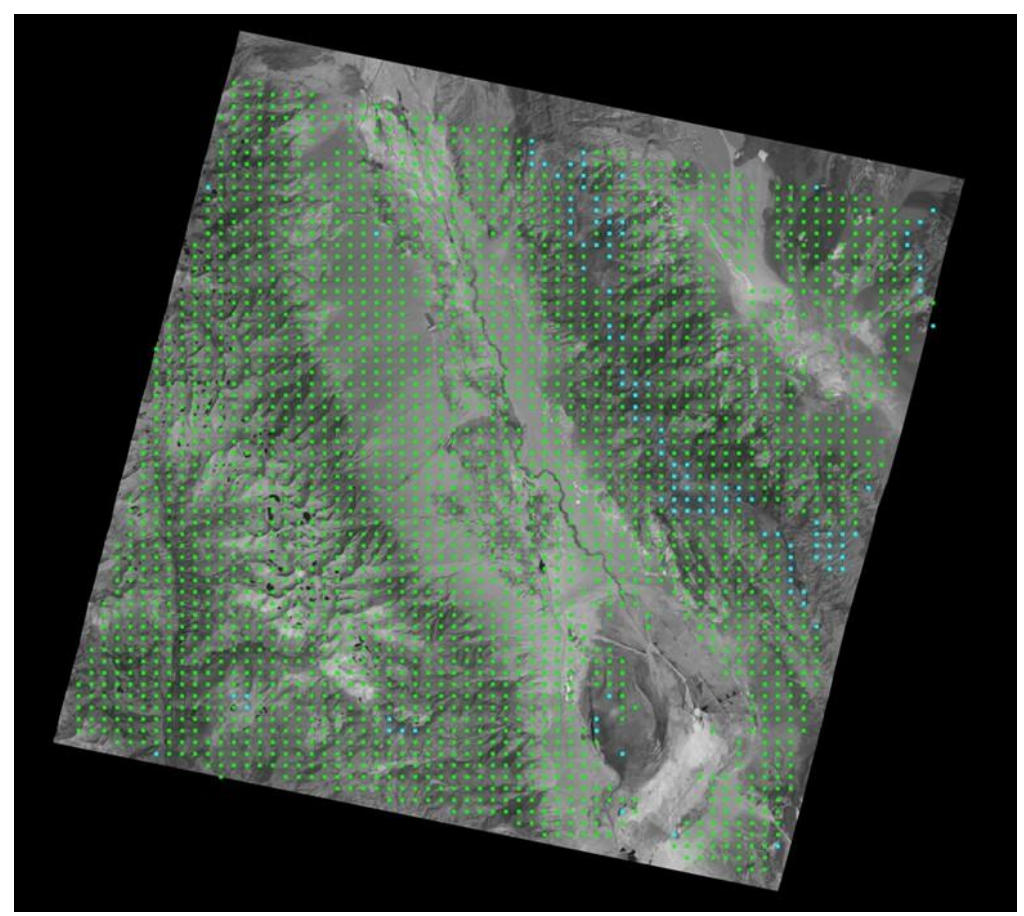

Figure 18. Example of browse image with valid GCP overlaid on an AST_L1T single band image.

The GVERIFY algorithm has the following known limitations associated with accurately correlating points from the terrain corrected AST_L1T granule to the reference GLS2000 image:

- The GLS2000 band $5(1.55-1.75 \mu \mathrm{m})$ is used as a reference, whereas band $4(1.60-1.70$ $\mu \mathrm{m})$ when available, or alternate band $2(0.63-0.69 \mu \mathrm{m})$ of the AST_L1T scene is used as a search image for correlation. Depending on band availability, the spectral range may have little or no overlap, and as a result may not provide accurate and consistent correlation peaks for different land features.

- The GLS2000 band 5 scene has a native spatial resolution of 30 meters, whereas the AST_L1T input bands are either 30 meters (band 4) or 15 meters (band 2). When the 15 meter band 2 was used, it is up sampled to 30 meters to match the resolution of the GLS2000 product for correlation to the GLS2000 image, which may result in poor contrast affecting the accuracy of correlations. 
- The temporal variation between the GLS2000 dataset generated from the Landsat Enhanced Thematic Mapper Plus (ETM+) sensor scenes acquired between 1999-2003, and the AST_L1T generated from recently acquired ASTER data may result in misregistration or the detection of temporal variations as true registration offsets.

- A GLS2000 scene must be available for the Worldwide Reference System (WRS) path, row of the AST_L1T scene center. For example, the GLS2000 data are not available for Antarctica.

- Correlation between the AST_L1T input band and the GLS2000 image may fail or produce false registration offsets because of cloud cover, and extreme terrain shadowing caused by sun angle.

\section{Full Resolution GeoTIFF Browse Images}

After the final resampling of the ASTER Level 1 input image described in the resample AST_L1A section, full resolution pseudo true color GeoTIFF composite images are generated from a subset of bands as determined in table 2, using the Geospatial Data Abstraction Library (GDAL) for inclusion in the final ASTER L1T deliverable product.

Table 2. Selected bands and pixel units of full resolution GeoTIFF browse images Band (B) and Nadir (N)

\begin{tabular}{|l|l|l|l|l|}
\hline \multicolumn{1}{|c|}{ Bands Acquired } & \multicolumn{1}{|c|}{ Red } & \multicolumn{1}{|c|}{ Green } & \multicolumn{1}{c|}{ Blue } & \multicolumn{1}{c|}{ Pixel Units } \\
\hline $\begin{array}{l}\text { Visible Infrared/Shortwave Infrared } \\
\text { (bands 1-9) }\end{array}$ & B4 & B3N & B2 & Reflectance \\
\hline Visible Infrared only (bands 1-3N) & B2 & B3N & B1 & Reflectance \\
\hline Thermal Infrared only (bands 10-14) & B14 & B12 & B10 & 8-bit scaled temperature factor \\
\hline
\end{tabular}

\section{Advanced Spaceborne Thermal Emission and Reflection Radiometer Level 1 Precision Terrain Corrected Registered At-Sensor Radiance Product}

The NASA LP DAAC produces the multifile AST_L1T product in collaboration with the U.S. Geological Survey reusing Landsat precision and terrain correction algorithms adapted for use with ASTER imagery. This GIS-ready product, generated from a single resample of the ASTER Level 1 input scene, includes 14 HDF-EOS4 bands of calibrated radiance data, fullresolution GeoTIFF browse image(s), and an associated XML metadata file. Additionally, the associated browse (thumbnail) images are available, and when created, associated quality assurance (QA) products. 


\section{References Cited}

Adelson, E.H., Anderson, C.H., Bergen, J.R., Burt, P.J., and Ogden, J.M., 1984, Pyramid methods in image processing: RCA Engineer, v. 29, no 6, p. 33-41.

Arai, Kohei, 2014, Vicarious calibration of Terra/ASTER/VNIR with desert scenes together with cross calibration in James J. Butler, Xiaoxiong (Jack) Xiong, Xingfa Gu, eds., SPIE Proceedings: Earth Observing Systems, v. 9218, no 19, p. 1-9, http://dx.doi.org/10.117/12.2060226.

Arai, Kohei, Tome, Kurtis, Iwasaki, Akira, and Bigger, Stuart, 2011a, ASTER VNIR and SWIR radiometric calibration and atmospheric correction in Ramachandran, Bhaskar Justice, C.O., and Abrams, M.J., eds., Land Remote Sensing and Global environmental change, remote sensing and digital image processing: Springer Science and Business Media, p. 83-116, http://dx.doi.org/10.1007/978-1-4419-6749-7.

Arai, Kohei, Ohgi, Nagamitsu, Sakuma, Fumihiro, Kikuchi, Masakuni, Tsuchida, Satoshi, and Inada, Hitomi, 2011b, Trend analysis of onboard calibration data of Terra/ASTER/VNIR and one of the suspected causes of sensitivity degradation, International Journal of Applied Science, v. 2, no. 3, p. 71-83.

Earth Remote Sensing Data Analysis Center, 1996, ASTER Algorithm Theoretical Basis Document for ASTER Level-1 Data Processing Version 3, By Level 1 Data Working Group, ASTER Science Team, ERSDAC LEL/8-9. Japan. (Also available at http://eospso.gsfc.nasa.gov/sites/default/files/atbd/atbd-ast-01.pdf)

Earth Remote Sensing Data Analysis Center, 2005, ASTER Release Notes, accessed July 16, 2014 at http://gds.aster.ersdac.jspacesystems.or.jp/gds_www2002/service_e/release_e/g.t.u.p9_e.html. Earth Remote Sensing Data Analysis Center, 2007, ASTER User's Guide Part II, Level 1 Data Products. Ver.5.1, accessed November 13, 2014 at http://www.science.aster.ersdac.jspacesystems.or.jp/en/documnts/users_guide/part1/pdf/Part2 _5.1E.pdf.

Iwasaki, Akira, and Tonooka, Hideyuki, 2005, Validation of a crosstalk correction algorithm for ASTER/SWIR, IEEE Transactions on Geoscience and Remote Sensing, v. 43, no. 12, p. 27472751.

Land Processes Distributed Active Archive, 2012, ASTER Level-1 Software Update, accessed October 27, 2014 at https://lpdaac.usgs.gov/about/news_archive/aster_level_1_software_update.

Park, Stephen K. and Schowengerdt, Robert A., 1983, Image reconstruction by parametric cubic convolution, Computer Vision, Graphics, and Image Processing, v. 23, no. 3, p. 258-272, http://dx.doi.org/10.1016/0734-189X(83)90026-9.

Rengarajan, Rajagopalan, Sampath, Aparajithan, Storey, James., and Choat, Michael., 2015, Validation of geometric accuracy of global land survey (gls) 2000 data, Photogrammetric Engineering \& Remote Sensing, v. 81, no. 2, p. 131-141, http://dx.doi.org/10.14358/PERS.81.2.131.

Thome, K., Biggar, S., and Choi., H. J., 2004, Vicarious Calibration of Terra ASTER, MISR, and MODIS in Barnes, William L. and Butler, James J., SPIE Proceedings: Earth Observing Systems, v. 5542, no. 9, p. 290-299, http://dx.doi.org/10.1117/12.559942.

Thome, Kurt J., Arai, Kohei, Tsuchida, Satoshi, Biggar, Stuart F., 2008, Vicarious calibration of ASTER via the reflectance-based approach, IEEE Transactions on Geoscience and Remote Sensing, v. 46, no. 10, p. 3285-3295, http://dx.doi.org/10.1109/TGRS.2008.928730. 
Tonooka Hideyuki and Iwasaki, Akira, 2004, Improvement of ASTER/SWIR crosstalk correction, Proceedings of SPIE, v. 5234, p. 168-179, http://dx.doi.org/10.1117/12.511811.

U.S. Geological Survey, 2006, Landsat 7 image assessment system (IAS) geometric algorithm theoretical basis document, Technical Report LS-IAS-01. Version 1.0. (Also available at http://landsat.usgs.gov/documents/LS-IAS-01_Geometric_ATBD.pdf)

U.S. Geological Survey, Earth Resource Observation and Science Center, 2008, Extraction of GCP chips from geo-cover using modified moravec interest operator (MMIO) algorithm, Technical Report. (Also available at http://landsat.usgs.gov/documents/MMIO_GeoCover_Control_White_Paper.pdf)

U.S. Geological Survey, 2012, Multispectral Scanner (MSS) Geometric Algorithm Description Document (ADD), Technical Report LS-IAS-06, Version 1.0. (Also available at http://landsat.usgs.gov/documents/LS-IAS-06.pdf) 


\title{
Appendix 1. ASTER Shortwave Infrared User Advisory July 18, 2008
}

\author{
Change in Status Alert - July 18, 2008
}

ASTER shortwave infrared (SWIR) bands continue to be adversely impacted by an anomalously high SWIR detector temperature. Although the ASTER team continues to focus on this situation, no improvement is anticipated in the next few weeks. Users are advised that ASTER SWIR data acquired from late April 2008, to the present exhibit anomalous saturation of values and anomalous striping. Cloud cover assessment and thermal infrared (TIR) location accuracy have also been affected. The data quality impacts referenced in the April 9, 2008, alert still apply for the periods specified. Earlier archived data conform to mission specifications.

Additional advisories will continue to be provided.

\section{Change in Status Alert - May 21, 2008}

As previously reported, the ASTER SWIR detector temperature rose precipitously on April 23, 2008, and SWIR data saturation occurred. The SWIR recycling procedure initiated on May 7, 2008, was not successful in lowering the SWIR detector temperature. Users are advised that ASTER SWIR data acquired from late April to the present exhibit anomalous saturation of values and anomalous striping. Cloud cover assessment and TIR location accuracy have also been affected. The data quality impacts referenced in the April 9, 2008, alert still apply for the periods specified. Earlier archived data conform to mission specifications.

The ASTER team is reviewing actions that might be taken in response to this situation. Additional advisories will continue to be provided.

\section{Change in Status Alert - May 2, 2008}

Users are advised that ASTER SWIR data acquired in late April, and early May 2008, exhibit anomalous saturation of values and anomalous striping. Cloud cover assessment and TIR location accuracy have also been affected by the present situation.

Since January 2008, SWIR performance has been stable and data quality has been nominal. On April 23, 2008, the SWIR detector temperature rose precipitously, and SWIR bands 5-9 saturated.

In an attempt to lower the SWIR detector temperature and improve data quality, the ASTER team plans to commence another SWIR recycling procedure on May 7, 2008. If successful, stable SWIR performance and nominal data quality will be restored. specified.

The data quality impacts referenced in the April 9, 2008, alert still apply for the periods

Additional advisories will continue to be provided.

\section{ASTER SWIR User Advisory - April 9, 2008}

This advisory is written to users of ASTER SWIR data to alert them to the fact that some anomalous saturation of values has been observed in ASTER bands 5 through 9 beginning May 2007. In addition, radiometric offset errors of up to 10 digital numbers (DNs) have been observed in these bands for data acquired between September 2007, and January 2008, resulting in noticeable imaging striping for some scenes. These problems are attributed to an increase in ASTER SWIR detector temperature believed to be caused by increased thermal resistance in the SWIR cryocooler. VNIR and TIR bands are unaffected by this problem. The slow increase in 
SWIR detector temperature, which gradually reduces the dynamic range of the SWIR bands, did not become a problem until early in 2007, and it did not really affect data quality until the detector temperature exceeded $83^{\circ} \mathrm{Kelvin}(\mathrm{K})$. The graph in figure 1 shows the trend in SWIR detector temperature for the past year. Note that the detector temperature first exceeded $83{ }^{\circ} \mathrm{K}$ on about May 1, 2007. Following that date, four attempts have been made to lower the detector temperature by recycling the cryocooler, including increasing the stroke length of the cryocooler piston.

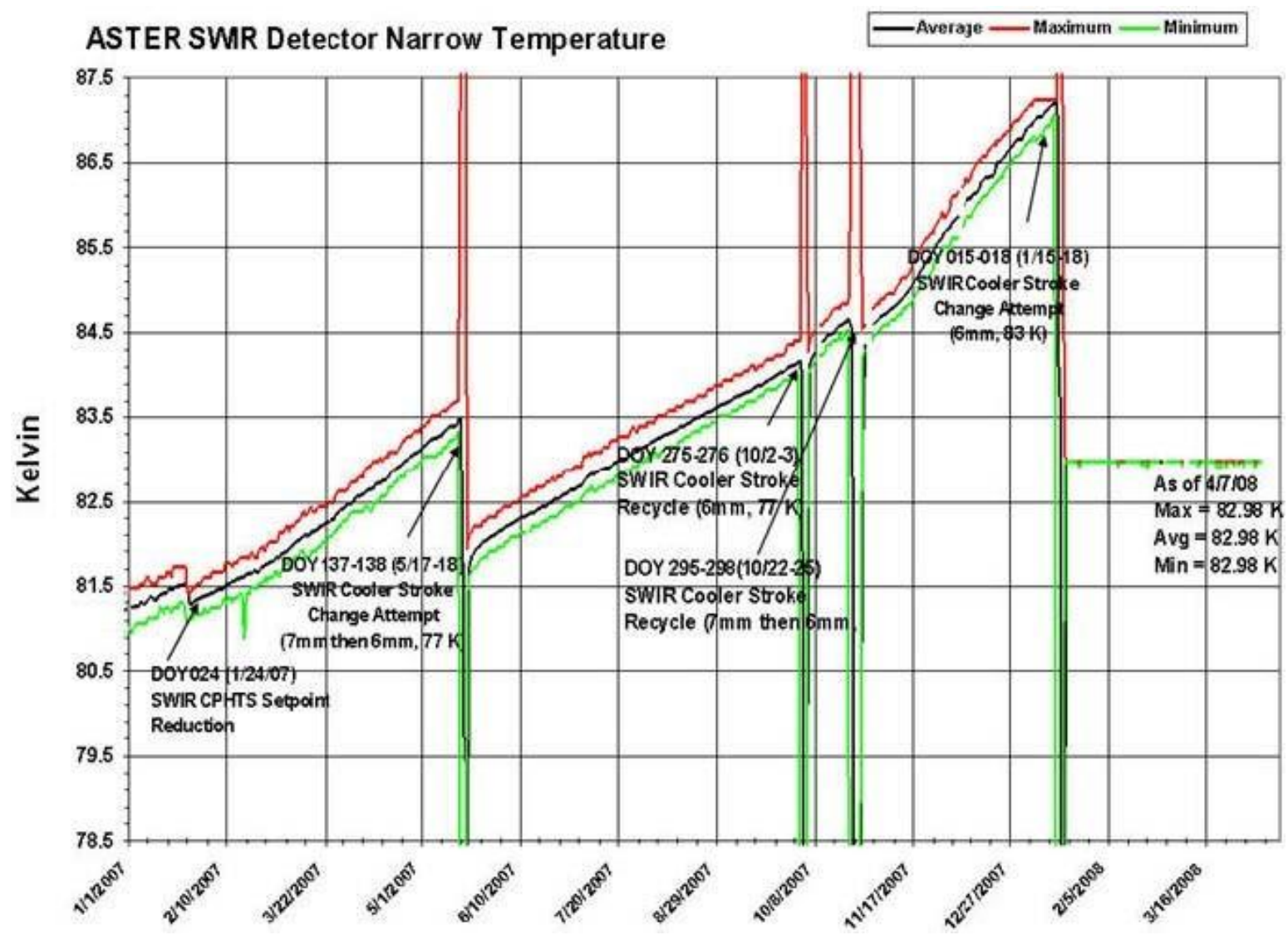

Figure 1. ASTER SWIR detector narrow temperature trend. Maximum (red), average (black), and minimum (green) lines are plotted.

The first attempt in May succeeded in reducing the temperature to $82^{\circ} \mathrm{K}$, but the temperature soon began to increase again, exceeding $83^{\circ} \mathrm{K}$ in late July. Second and third attempts to reduce the SWIR detector temperature essentially failed in October. However, a fourth attempt in early January 2008 succeeded in reducing the SWIR detector temperature to 83 ${ }^{\circ} \mathrm{K}$. Since that date the SWIR detector temperature has remained stable at $83^{\circ} \mathrm{K}$.

As long as the detector temperature remains at $83^{\circ} \mathrm{K}$, little or no degradation of ASTER SWIR data is expected. However, users are advised that for ASTER SWIR data acquired between late May 2007 and late January 2008, the SWIR detector temperature exceeded $83{ }^{\circ} \mathrm{K}$, except for about 6 weeks in June and July. SWIR data acquired during these periods may exhibit anomalous saturation of values, particularly at high sun angles and for materials that are highly reflective in the SWIR bands. SWIR data acquired between September 2007 (when the detector 
temperature first exceeded $84^{\circ} \mathrm{K}$ ) and January 2008, have radiometric offset errors that exceed 5 $\mathrm{DN}$, and the corresponding image data may exhibit anomalous striping.

An example of SWIR saturation in an extremely bright desert scene acquired over northern Africa in August 2007, when the detector temperature was at about $83.5^{\circ} \mathrm{K}$, is shown in figure 2. Saturation is especially prevalent in bands 5, 6, and 7. Saturated pixels with DN $=255$ are displayed in black. All other colors are unsaturated pixels.

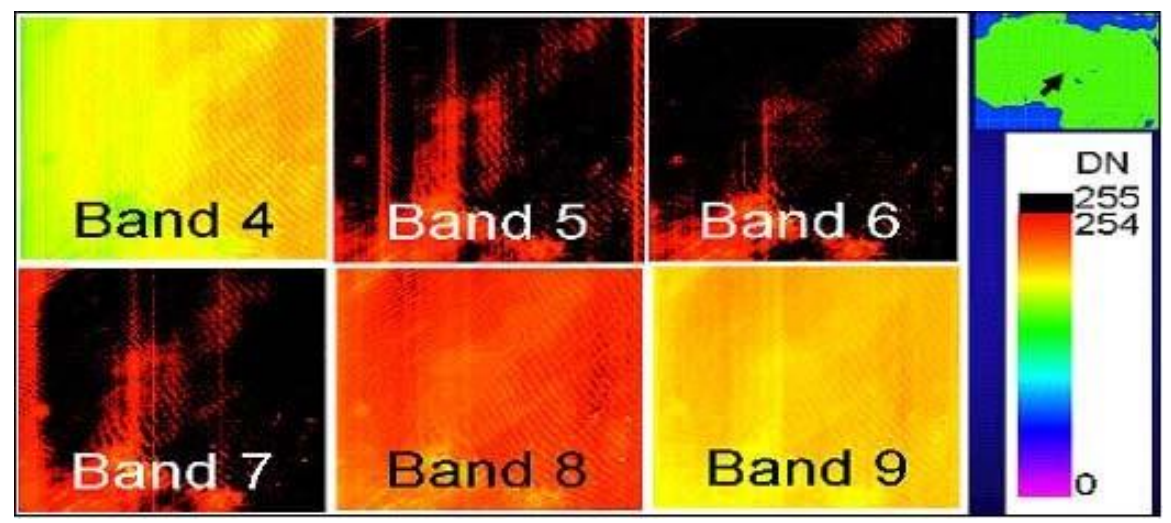

Figure 2. SWIR saturation example. August 24, 2007, Africa observation

An example of image striping that results from the radiometric offset error described above is shown in figure $3 A$. While it is not possible to apply any correction to reverse the image saturation anomalies in the SWIR, it is possible to correct the radiometric offset errors and eliminate the anomalous image striping. The effects of applying updated radiometric correction coefficients to the SWIR data collected when the detector temperature exceeded $84^{\circ} \mathrm{K}$ is shown in figure $3 B$.

ASTER Ground Data System (GDS) has initiated a 6-month effort to reprocess ASTER data collected between September 2007 and January 2008, when the detector temperature exceeded $84^{\circ} \mathrm{K}$. The LPDAAC will sequentially replace existing data acquired during this period with data newly corrected for radiometric offset and with anomalous striping removed as they are received from ASTER GDS.

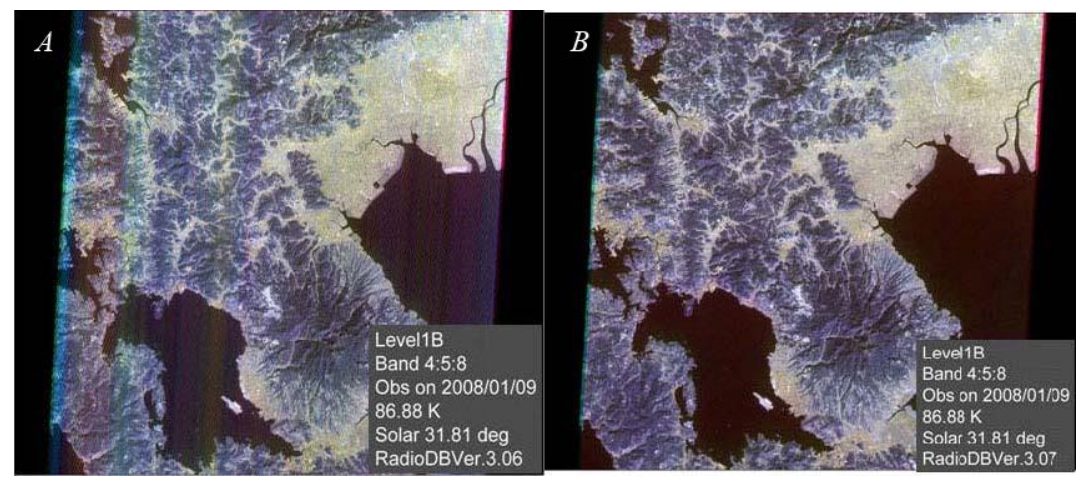

Figure 3. Image $(A)$ with striping, which results from radiometric offset errors in SWIR data caused by increasing detector temperature compared with the same image $(B)$ with striping removed by application of updated radiometric correction coefficients. Striping does not become readily apparent in current data until detector temperatures exceed $84^{\circ} \mathrm{K}$. Reprocessing of data acquired when detector temperatures exceeded $84{ }^{\circ} \mathrm{K}$ will result in an archive of SWIR data optimally corrected for radiometric offset errors. 